\title{
Optimal piezoelectric actuator and sensor location for active vibration control, using genetic algorithm
}

\author{
Isabelle Bruant*, Laurent Gallimard, Shahram Nikoukar \\ Laboratoire Energétique, Mécanique, Electromagnétisme, EA 4416, Université Paris Ouest Nanterre La Défense, 50 rue de Sèvres, 92410 Ville d'Avray, France
}

\begin{abstract}
A B S T R A C T
This paper deals with the optimization of piezoelectric actuators and sensors locations for active vibration control. Two modified optimization criteria are used, ensuring good observability or controllability of the structure, and considering residual modes to limit the spillover effect. Two optimization variables are considered for each piezoelectric device: the location of its center and its orientation. Genetic algorithms are used to find the optimal configurations. Several simulations are presented for a simply supported plate.
\end{abstract}

\section{Introduction}

In recent years, a great number of research results has been produced in active vibration control of flexible structures using piezoelectric actuators and sensors. It is obvious that misplaced sensors and actuators lead to problems such as the lack of observability and controllability which decreases strongly the performance of the control system. Many papers dealing with the optimization of actuators and sensors location can be found in the scientific journals. An exhaustive review until 2001 is presented in [1].

Two approaches can be distinguished. The first one consists of combining optimization of actuators/sensors locations and controller parameters. For example [2-7] propose a quadratic cost function taking into account the measurement error and the control energy. In [8], the energy dissipation method has been adopted as the criterion for the optimization of the control system. This method is based on the maximization of dissipation energy due to the control action. In [9], the spatial $H 2$ norm of the closed-loop transfer matrix from the disturbance to the distributed controlled output is used as the optimization index. Refs. [10,11] suggest the simultaneous design of a computationally simple $H \infty$ controller and optimization of the location of sensors and actuators. In this first approach, the optimization criteria are dependent on the choice of controllers. Therefore, the optimal locations obtained using one controller may not be a suitable choice for another one.

In the second approach, the optimal locations are obtained independently of the controller definition. Several cost functions are used. Refs. [12-15,17,18] propose the maximization of a controllability/observability criterion using the gramian matrices. Ref. [19] suggests the maximization of the control forces transmitted by the actuators to the structure. Ref. [20] proposes a modal controllability index based on the same singular value analysis of the control vector. In [21-23], an optimal placement method using $\mathrm{H} 2$ norm is presented.

\footnotetext{
* Corresponding author.

E-mail address: isabelle.bruant@u-paris10.fr (I. Bruant).
} 
The spillover effects are a significant problem of active control implementation on real structures. As a matter of fact, because of the truncation of the model, there is no guarantee that the higher frequency modes will not contribute to the control spillover if a feedback controller is implemented on the system, since this controller will unintentionally excite them. However, few papers take into account the residual modes in the optimization problem, see [17,23-25].

In this paper, in order to simplify the optimization problem, and not to be limited to collocated actuators and sensors, the optimal location of sensors and actuators are computed independently. The modified optimization criteria presented in [17] are used. They ensure good observability and good controllability of the structures and consider residual modes to limit the spillover effects. The optimization parameters are the location of the actuator/sensor center and their orientation on the structure. A numerical approach using a Genetic Algorithm (GA) method [26] is adopted to maximize the two cost functions. GA methods are computationally effective in finding the global optimal solution for a not convex function which has not derivative. Several authors have yet used this method to optimize the actuators and sensors locations, for example $[7-9,15,18,16]$.

In the first part of this work, we point out the active vibration control equations. In the third section, the optimization criteria used for sensors and actuators locations are presented. Then, the GA method is briefly recalled in Section 4 . Results are shown for a simply supported plate in Section 5. Finally, Section 6 is devoted to the application of the GA to active vibration control.

\section{Equations of active vibration control}

Consider a flexible structure with $N_{a}$ piezoelectric actuators and $N_{s}$ piezoelectric sensors. From analytical model or finite element analysis, equations of motion and the sensors' output equations of the system in modal coordinates can be written as follows:

$$
\begin{gathered}
\ddot{\alpha}_{i}+2 \zeta_{i} \omega_{i} \dot{\alpha}_{i}+\omega_{i}^{2} \alpha_{i}=\sum_{l=1}^{N_{a}} b_{i l} \Phi_{l}, \quad i=1, \ldots, N \\
\ddot{\alpha}_{i}+2 \zeta_{i}^{R} \omega_{i}^{R} \dot{\alpha}_{i}+\left(\omega_{i}^{R}\right)^{2} \alpha_{i}=\sum_{l=1}^{N_{a}} b_{i l}^{R} \Phi_{l}, \quad i=1, \ldots, N^{R} \\
y_{j}=\sum_{l=1}^{N} c_{j l} \alpha_{l}+\sum_{l=1}^{N^{R}} c_{j l}^{R} \alpha_{l}, \quad j=1, \ldots, N_{s}
\end{gathered}
$$

where the $N$ first eigenmodes and $N^{R}$ residual eigenmodes are considered. The choice of $N$ and $N^{R}$ depends on the external loads applied to the structure. $\alpha_{i}, \dot{\alpha}_{i}$ and $\ddot{\alpha}_{i}$ represent modal displacement, velocity and acceleration, $\omega_{i}$ and $\zeta_{i}$ are the natural frequency and damping ratio of the $i$ th mode, and $\omega_{i}^{R}$ and $\zeta_{i}^{R}$ those of the residual modes; $b_{i l} \Phi_{l}$ is the $i$ th modal component of the control force due to the electric potential $\Phi_{l}$ applied to the $l$ th actuator, $b_{i l}^{R} \Phi_{l}$ is the $i$ th residual modal component of the force appearing with the actuation of the actuator $l . y_{j}$ is the quantity measured from the $j$ th sensor. $c_{j l}$ is the sensing constant of the $j$ th sensor due to the motion of the $l$ th mode and $c_{j l}^{R}$ those due to the motion of the $l$ th residual mode. $b_{i l}, b_{i l}^{R}, c_{j l}, c_{j l}^{R}$ depend respectively, on the $l$ th actuator location and $j$ th sensor location.

These equations can be written in an usual state-space form, using the state vector $\{\mathbf{x}\}$ (size $\left.\left(N+N^{R}\right)+\left(N+N^{R}\right)\right)$ :

$$
\begin{gathered}
\{\mathbf{X}\}=\left\{\omega_{i} \alpha_{i} \dot{\alpha}_{i}\right\}^{\mathrm{T}} \\
\frac{\mathrm{d}}{\mathrm{d} t}\{\mathbf{X}\}=[\mathbf{A}]\{\mathbf{X}\}+[\mathbf{B}]\{\mathbf{\Phi}\} \\
\{\mathbf{y}\}=[\mathbf{C}]\{\mathbf{x}\}
\end{gathered}
$$

where $[\mathbf{A}]_{\left(2 N+2 N^{R}, 2 N+2 N^{R}\right)},[\mathbf{B}]_{\left(2 N+2 N^{R}, N_{a}\right)}$ and $[\mathbf{C}]_{\left(N_{s}, 2 N+2 N^{R}\right)}$ are the state, control and output matrices given by

$$
\begin{gathered}
{[\mathbf{A}]=\left[\begin{array}{cccc}
{[0]} & {[0]} & {\left[\omega_{i}\right]} & {[0]} \\
{[0]} & {[0]} & {[0]} & {\left[\omega_{i}^{R}\right]} \\
-\left[\omega_{i}\right] & {[0]} & -\left[2 \zeta_{i} \omega_{i}\right] & {[0]} \\
{[0]} & -\left[\omega_{i}^{R}\right] & {[0]} & -\left[2 \zeta_{i}^{R} \omega_{i}^{R}\right]
\end{array}\right]} \\
{[\mathbf{B}]^{\mathrm{T}}=\left[[0]\left[b_{i l}\right][0]\left[b_{i l}^{R}\right]\right]} \\
{[\mathbf{C}]=\left[\left[c_{j l}\right]\left[c_{j l}^{R}\right][0][0]\right]}
\end{gathered}
$$

$\{\boldsymbol{\Phi}\}$ is the electric potential vector applied to the piezoelectric actuators.

From Eq. (5), several automatic tools can be used to actively control vibrations [27]. Even as the actuation must be designed to bend the $N$ first eigenmodes, it also excites the residual modes. This effect is called spillover [27]. In fact, the 
best case would be having

$$
\begin{gathered}
b_{i l} \gg 0, \quad \forall l \in 1, \ldots, N_{a}, \quad \forall i \in 1, \ldots, N \\
b_{i l}^{R}=0, \quad \forall l \in 1, \ldots, N_{a}, \quad \forall i \in 1, \ldots, N^{R},
\end{gathered}
$$

ensuring actuation (or controllability) for the $N$ first modes and no influence (or no controllability) of the $N^{R}$ residual modes.

In the same way, the vibrational information given by the piezoelectric sensors to the control system contains contributions on residual modes. As these residual modes are neglected in the control system, the sensors information does not correspond to the required information. The best case would be having

$$
\begin{gathered}
c_{j l} \gg 0, \quad \forall j \in 1, \ldots, N_{s}, \quad \forall l \in 1, \ldots, N \\
c_{j l}^{R}=0, \quad \forall j \in 1, \ldots, N_{s}, \quad \forall l \in 1, \ldots, N^{R}
\end{gathered}
$$

in order to have each mode $(l \in 1, \ldots, N)$ to be well observed (good observability) and each residual mode not to be observed (non-observability).

Hence, before setting up the regulator and observer system, the active elements' locations have to be defined.

\section{The optimization criteria for piezoelectric actuators and sensors locations}

In this paper, the modified optimization criteria presented in [17] are used. They ensure good observability and good controllability of each mode considering them with homogeneity and not globally as it is usually done. The residual modes are taken into account to limit the spillover effects.

\subsection{Optimal location of sensors}

When the system is released from the initial state $\{\mathbf{x}(0)\}=\left\{\mathbf{x}_{0}\right\}$, as when it is subjected to a persistent disturbance, [13] has shown that maximizing the system output $\int_{0}^{\infty}\{\mathbf{y}\}^{\mathrm{T}}\{\mathbf{y}\} \mathrm{d} t$ (for desired modes) yields maximizing the gramian observability matrix defined by

$$
\left[\mathbf{W}_{o}\right]=\int_{0}^{\infty} \mathrm{e}^{[\mathbf{A}]^{\mathrm{T}} t}[\mathbf{C}]^{\mathrm{T}}[\mathbf{C}] \mathrm{e}^{[\mathbf{A}] t} \mathrm{~d} t
$$

where $\mathbf{W}_{o}$ tends to a diagonal form

$$
\left(\mathbf{W}_{o}\right)_{i i}=\left(\mathbf{W}_{o}\right)_{i+N, i+N}=\sum_{j=1}^{N_{s}} \frac{c_{j i}^{2}}{4 \zeta_{i} \omega_{i}}=\frac{1}{4 \zeta_{i} \omega_{i}} \sum_{j=1}^{N_{s}} c_{j i}^{2}, \quad i=1, \ldots, N
$$

Each diagonal term $\left(\mathbf{W}_{o}\right)_{i i}$ corresponds to the maximization of the output energy $J_{i}$ for the $i$ th mode obtained if we consider the state equation reduced to the $i$ th mode:

$$
\begin{aligned}
{\left[\begin{array}{c}
\omega_{i} \dot{\alpha}_{i} \\
\ddot{\alpha}_{i}
\end{array}\right] } & =\left[\begin{array}{cc}
0 & \omega_{i} \\
-\omega_{i} & -2 \zeta_{i} \omega_{i}
\end{array}\right]\left[\begin{array}{c}
\omega_{i} \alpha_{i} \\
\dot{\alpha}_{i}
\end{array}\right]+\left[\begin{array}{c}
{[0]} \\
{\left[b_{i l}\right]}
\end{array}\right]\{\boldsymbol{\Phi}\} \\
\{\mathbf{y}\} & =\left[C_{i}\right]\left[\begin{array}{c}
\omega_{i} \alpha_{i} \\
\dot{\alpha}_{i}
\end{array}\right], \quad J_{i}=\int_{0}^{\infty}\{\mathbf{y}\}^{\mathrm{T}}\{\mathbf{y}\} \mathrm{d} t
\end{aligned}
$$

Consequently, if the $i$ th eigenvalue of $\mathbf{W}_{o}$ is small, it means that the $i$ th mode will not be observed well.

To have a convenient information about the $N$ first eigenmodes and to minimize the information of each residual mode, the following optimization criterion could be considered: find the sensors locations $S_{1}, \ldots, S_{N_{s}}$ to maximize

$$
\left\{\min _{i=1, \ldots, N}\left(\mathbf{W}_{o}^{U}\left(S_{1}, \ldots, S_{N_{s}}\right)\right)_{i i}-\gamma \max _{i=1, \ldots, N^{R}}\left(\mathbf{W}_{o}^{R}\left(S_{1}, \ldots, S_{N_{s}}\right)\right)_{i i}\right\}
$$

where $\gamma$ is a weighting constant. $\left(\mathbf{W}_{o}^{U}\left(S_{1}, \ldots, S_{N_{s}}\right)\right)_{i i}$ and $\left(\mathbf{W}_{o}^{R}\left(S_{1}, \ldots, S_{N_{s}}\right)\right)_{i i}$ are respectively, the output energy of the $i$ th unresidual and the $i$ th residual eigenmode, when the $N_{s}$ sensors are located in $S_{1}, \ldots, S_{N_{s}}$. But as the components of $\mathbf{W}_{o}$ have not the same range, solving this problem can induce the study of particular modes instead of each of them, and then the obtained locations will not be optimal. Hence, to establish homogeneity between each term $\left(\mathbf{W}_{o}\right)_{i i}$ we have suggested in [17] to divide each of them by its maximal value obtained when the $i$ th mode is the specific mode to be measured. Then, the optimization problem, considered here is to find the sensors locations $S_{1}, \ldots, S_{N_{s}}$ which maximize

$$
J_{S}=\min _{i=1, N} \frac{\left(\mathbf{W}_{o}^{U}\left(S_{1}, \ldots, S_{N_{s}}\right)\right)_{i i}}{\max _{S_{1}, \ldots, S_{N_{s}}}\left(\mathbf{W}_{o}^{U}\left(S_{1}, \ldots, S_{N_{s}}\right)\right)_{i i}}-\gamma \max _{i=1, N^{R}} \frac{\left(\mathbf{W}_{o}^{R}\left(S_{1}, \ldots, S_{N_{s}}\right)\right)_{i i}}{\max _{S_{1}, \ldots, S_{N_{s}}}\left(\mathbf{W}_{o}^{R}\left(S_{1}, \ldots, S_{N_{s}}\right)\right)_{i i}}
$$




$$
\begin{gathered}
=\min _{i=1, N} \frac{\sum_{j=1}^{N_{s}} c_{j i}^{2}}{\max _{S_{1}, \ldots, S_{N_{s}}} \sum_{j=1}^{N_{s}} c_{j i}^{2}}-\gamma_{i=1, N^{R}} \frac{\sum_{j=1}^{N_{s}}\left(c_{j i}^{R}\right)^{2}}{\max _{S_{1}, \ldots, S_{N_{s}}} \sum_{j=1}^{N_{s}}\left(c_{j i}^{R}\right)^{2}} \\
\text { with } \quad \forall i=1, \ldots, N+N^{R}, \quad 0 \leq \frac{\left(\mathbf{W}_{o}\left(S_{1}, \ldots, S_{N_{s}}\right)\right)_{i i}}{\max _{S_{1}, \ldots, S_{N_{s}}}\left(\mathbf{W}_{o}\left(S_{1}, \ldots, S_{N_{s}}\right)\right)_{i i}} \leq 1
\end{gathered}
$$

In this approach, all the modes are studied with the same range. $\max _{S_{1}, \ldots, S_{N_{s}}}\left(\mathbf{W}_{o}\left(S_{1}, \ldots, S_{N_{s}}\right)\right)_{i i}$ represents the maximal output energy which could be measured for the $i$ th mode by the sensors. Residual modes are not neglected; their influence on the structure dynamic is controlled by using $\gamma$.

\subsection{Optimal location of actuators}

The optimal locations of actuators are determined in the same way as the optimal locations of sensors. The objective here is to find actuators locations that minimize the control energy required to bring the modal system (considering the $N$ first eigenmodes) to a desired state $\left\{\mathbf{x}_{T}\right\}$ after some time $T$ :

$$
J=\min _{\{\boldsymbol{\Phi}\}} \int_{0}^{T}\{\boldsymbol{\Phi}\}^{\mathrm{T}}\{\boldsymbol{\Phi}\} \mathrm{d} t
$$

The optimal solution gives the following optimal control energy:

$$
J=\left[\mathrm{e}^{[\mathbf{A}]^{\mathrm{T}}}\left\{\mathbf{x}_{0}\right\}-\left\{\mathbf{x}_{T}\right\}\right]^{\mathrm{T}} \mathbf{W}^{-1}(T)\left[\mathrm{e}^{[\mathbf{A}]^{\mathrm{T}}}\left\{\mathbf{x}_{0}\right\}-\left\{\mathbf{x}_{T}\right\}\right]
$$

where $\mathbf{W}(T)$ is the controllability gramian matrix defined by

$$
[\mathbf{W}(T)]=\int_{0}^{T} \mathrm{e}^{[\mathbf{A}] t}[\mathbf{B}][\mathbf{B}]^{\mathrm{T}} \mathrm{e}^{[\mathbf{A}]^{\mathrm{T}} t} \mathrm{~d} t
$$

Minimizing $J$ with respect to the actuators locations consists in minimizing $\mathbf{W}^{-1}(T)$ or maximizing a measure of the controllability gramian matrix [13].

Ref. [13] has shown that instead of using $\mathbf{W}(T)$, a steady-state $\mathbf{W}_{c}$ can be considered to eliminate the dependency of the solution $T$. $\mathbf{W}_{c}$ tends to a diagonal form with

- for unresidual modes $(i=1, \ldots, N)$

$$
\left(\mathbf{W}_{c}\right)_{i i}=\left(\mathbf{W}_{c}\right)_{i+N+N^{R}, i+N+N^{R}}=\left(\mathbf{W}_{c}^{U}\right)_{i i}=\sum_{j=1}^{N_{a}} \frac{b_{i j}^{2}}{4 \zeta_{i} \omega_{i}}=\frac{1}{4 \zeta_{i} \omega_{i}} \sum_{j=1}^{N_{a}} b_{i j}^{2}
$$

- for residual modes $\left(i=1, \ldots, N^{R}\right)$

$$
\left(\mathbf{W}_{c}\right)_{i+N, i+N}=\left(\mathbf{W}_{c}\right)_{i+2 N+N^{R}, i+2 N+N^{R}}=\left(\mathbf{W}_{c}^{R}\right)_{i i}=\frac{1}{4 \zeta_{i}^{R} \omega_{i}^{R}} \sum_{j=1}^{N_{a}}\left(b_{i j}^{R}\right)^{2}
$$

$\left(\mathbf{W}_{c}^{U}\right)_{i i}$ and $\left(\mathbf{W}_{c}^{R}\right)_{i i}$ equal to the energy transmitted from the actuators to the structure for the $i$ th used or residual eigenmode.

Hence, if the eigenvalue $\left(\mathbf{W}_{c}^{U}\right)_{i i}$ is small, the $i$ th eigenmode is difficult to control: there is no controllability for the system. Similarly, if the eigenvalue $\left(\mathbf{W}_{c}\right)_{i i}^{R}$ corresponding to the $i$ th residual mode is high, the induced spillover effect can be important.

The usual criteria take into account globally the eigenmode. Instead of maximizing a global norm of $\mathbf{W}_{c}$ which means minimizing the electrical energy, a first optimization criterion could be to find the actuators location which maximize

$$
\left\{\min _{i=1, \ldots, N}\left(\mathbf{W}_{c}^{U}\left(A_{1}, \ldots, A_{N_{a}}\right)\right)_{i i}-\tilde{\gamma}_{i=1, \ldots, N^{R}} \max _{c}\left(\mathbf{W}_{c}^{R}\left(A_{1}, \ldots, A_{N_{a}}\right)\right)_{i i}\right\}
$$

where $\tilde{\gamma}$ is a weighting constant. $A_{i}$ is the location of the $i$ th actuator. But as the components of $\mathbf{W}_{c}$ have not the same range, solving this problem can induce the study of particular modes instead of each of them, and then the obtained locations will not be optimal.

Consequently, each term $\left(\mathbf{W}_{c}\right)_{i i}$ is divided by its maximal value obtained when the $i$ th mode is the specific mode to be controlled. This maximal value is the maximal energy which can be transmitted from the actuators for the $i$ th eigenmode.

Hence, using the homogeneous components, the optimization problem becomes to find the actuators location which maximize

$$
J_{A}=\left\{\min _{i=1, N} \frac{\left(\mathbf{W}_{c}^{U}\left(A_{1}, \ldots, A_{N_{a}}\right)\right)_{i i}}{\max _{A_{1}, \ldots, A_{N_{a}}}\left(\mathbf{W}_{c}^{U}\left(A_{1}, \ldots, A_{N_{a}}\right)\right)_{i i}}-\tilde{\gamma} \max _{i=1, N^{R}} \frac{\left(\mathbf{W}_{c}^{R}\left(A_{1}, \ldots, A_{N_{a}}\right)\right)_{i i}}{\max _{A_{1}, \ldots, A_{N_{a}}}\left(\mathbf{W}_{c}^{R}\left(A_{1}, \ldots, A_{N_{a}}\right)\right)_{i i}}\right\}
$$




$$
=\min _{i=1, N} \frac{\sum_{j=1}^{N_{a}} b_{i j}^{2}}{\max _{A_{1}, \ldots, A_{N_{a}}} \sum_{j=1}^{N_{a}} b_{i j}^{2}}-\tilde{\gamma} \max _{i=1, N^{R}} \frac{\sum_{j=1}^{N_{a}}\left(b_{i j}^{R}\right)^{2}}{\max _{A_{1}, \ldots, A_{N_{a}}} \sum_{j=1}^{N_{a}}\left(b_{i j}^{R}\right)^{2}}
$$

and, $\forall i=1, \ldots, N+N^{R} \quad 0 \leq \frac{\left(\mathbf{W}_{c}\left(A_{1}, \ldots, A_{N_{a}}\right)\right)_{i i}}{\max _{A_{1}, \ldots, A_{N_{a}}}\left(\mathbf{W}_{c}\left(A_{1}, \ldots, A_{N_{a}}\right)\right)_{i i}} \leq 1$

The greatest advantage of this criterion is that all modes are studied with the same range. Residual modes again, are not neglected and their influence on the structure dynamic can be more or less important using $\tilde{\gamma}$. Furthermore, the expression inside (24) has a physical meaning: it is the mechanical energy transmitted for the $i$ th mode divided by the maximal mechanical energy that could be received.

\section{Optimization implementation using genetic algorithms}

The optimization problems, considered in this paper, are described in (16) and (24) where the numbers of actuators and sensors are fixed. As the optimization problem is not convex and the objective functions and constraints do not have closed form derivatives with respect to the design variables, genetic algorithms (GA) seem to be adapted well [26]. Several authors have yet used them for optimal location of actuators and sensors, see for example [7-9,15,18,28,29].

In this work, the two optimization problems are similar, but to simplify the optimization procedure, they are solved independently. Very few papers deal with the orientations of sensors and actuators [22,30]. Here, they are taken into account as optimization variables.

In this section, the use of GA for actuators location optimization is detailed. The optimization procedure for sensors locations is computed in the same way.

Finding the location of $N_{a}$ actuators, consists here of determining the coordinate of each actuator's center $\left(x^{a}, y^{a}\right)$ and the actuator's orientation $\left(0 \leq \theta^{a}<\pi\right)$. The optimization variables vector for optimal location of actuators is $\left\{\left(x_{1}^{a}, y_{1}^{a}, \theta_{1}^{a}\right),\left(x_{2}^{a}, y_{2}^{a}, \theta_{2}^{a}\right), \ldots,\left(x_{N_{a}}^{a}, y_{N_{a}}^{a}, \theta_{N_{a}}^{a}\right)\right\}$.

GAs are derived from the mechanics of natural selection and genetics. They are an effective numerical method to find an optimal (or sub-optimal) solution to a complicated multiparameter optimization problem, without calculating the derivatives of the function to be optimized. Basically, GA find the optimal solution through iterating the GA operations on a population, which consists of a number $N_{i}$ of candidate solutions to the optimization problem.

Vocabulary of natural genetics is used in GA:

- The considered population contains $N_{i}$ individuals.

- One individual represents a candidate solution of the optimization problem. In our work, it consists of the set of $N_{a}$ actuators. Each individual is defined by $N_{a}$ chromosomes.

- A chromosome here is the location of one actuator.

- Each chromosome is defined with a sequence of three genes: the coordinates of the actuator's center and the actuator's orientation.

Several representation for genes can be used (binary or real-encoded). Here, we use the real-encoded GA [31].

The GA method starts with a randomly generated population. At each iteration, a new population is created by repeating the following steps:

- Selection: select two parent individuals from the population according to their fitness value (i.e. $J_{A}$ criteria): the better fitness, the bigger chance to be selected.

- Crossover: using arithmetical crossover [31], the two selected parents give two children.

- Mutation: with a mutation probability, some chromosomes of the child are changed randomly.

- Place new offspring in the new population.

The crossover makes the GA process move in a desirable direction, and the mutation helps to prevent the process from getting trapped in a local optimal solution.

A conservation step is added and applied to the new generation: it consists of keeping the best parent (i.e. the greatest fitness value) in this new generation.

The GA stops when a certain number of iterations has been reached. Of course, the results obtained from GA process with the limited number of iterations might be a suboptimal solution. To get a result with higher confidence, one has to run the GA process either several times, each with a randomly generated initial conditions, or with sufficient number of iterations [9].

\section{Application of the GA for optimal location of piezoelectric devices}

In this section, the application of the optimization process is discussed. A simply supported elastic plate is considered, equipped with piezoelectric actuators and sensors. In order to consider only pure bending motion, each actuator and 
Table 1

Geometrical characteristics of the plate and the piezoelectric patch.

\begin{tabular}{llll}
\hline & Plate & Piezoelectric (tests 1-4, 6) & Piezoelectric (test 5) \\
\hline Length (m) & 0.38 & 0.02 & 0.073 \\
Width (m) & 0.3 & 0.01 & 0.016 \\
Thickness (m) & 0.002 & 0.0001 & 0.0002 \\
\hline
\end{tabular}

Table 2

Characteristics of piezoelectric patch PZT5A.

\begin{tabular}{lr}
\hline$\varepsilon_{33}\left(F \mathrm{~m}^{-1}\right)$ & $1.5 \mathrm{e}^{-8}$ \\
$e_{31}\left(C \mathrm{~m}^{-2}\right)$ & -7.209 \\
$e_{32}\left(C \mathrm{~m}^{-2}\right)$ & -7.209
\end{tabular}

Table 3

Mechanical characteristics of the elastic plate.

\begin{tabular}{ll}
\hline$\rho\left(\mathrm{kg} \mathrm{m}^{-3}\right)$ & 7870 \\
$E(\mathrm{GPa})$ & 207 \\
$v$ & 0.292 \\
$\zeta_{i}$ & 0.0001 \\
\hline
\end{tabular}

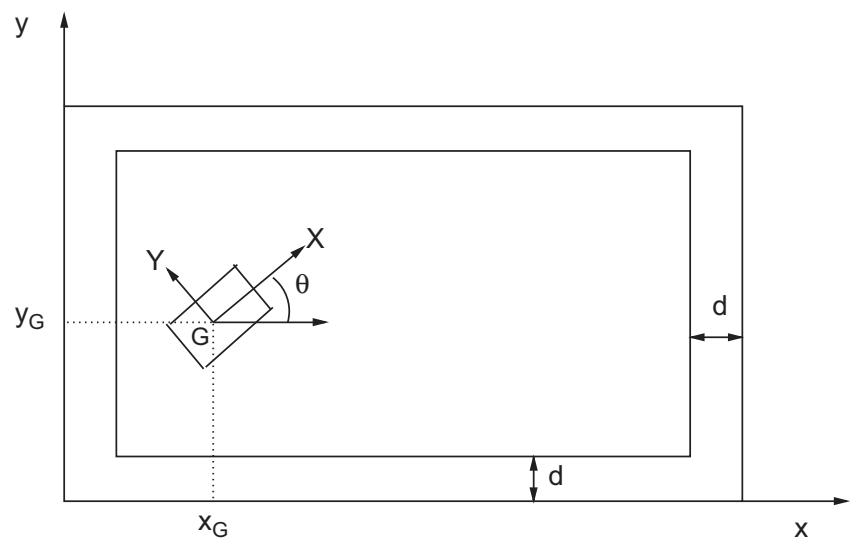

Fig. 1. An elastic plate equipped with one piezoelectric device.

sensor is made up of a pair of same piezoelectric material attached symmetrically. They are assumed to be perfectly bonded to the surface of the plate, and their thickness is assumed to be small compared to the plate thickness. The geometrical and mechanical properties of the system are detailed in Tables 1-3. The optimization variables considered in the optimization problems for one device are the coordinates of its center $G$ and its orientation (Fig. 1). $L, l, 2 h$ and $L_{p}, l_{p}$ and $h_{p}$ are respectively, the length, width, thickness of the plate and those of the piezoelectric patches.

\subsection{Analytical equations}

As the geometrical properties of piezoelectric are small compared to those of the elastic plate, piezoelectric patches can be neglected in the computation of eigenmodes. In case of an isotropic material for a simply supported plate, the analytical formula of eigenvalues and eigenmodes are well known and equal to

$$
\begin{aligned}
\psi_{r n}(x, y) & =\sin \left(\frac{r \pi x}{L}\right) \sin \left(\frac{n \pi y}{l}\right) \frac{2}{\sqrt{L l m}} \\
\omega_{r n} & =\pi^{2}\left(\left(\frac{r}{L}\right)^{2}+\left(\frac{n}{l}\right)^{2}\right) \sqrt{\frac{D}{m}}
\end{aligned}
$$

where $r$ and $n$ are the number of half waves in the $x$ and $y$ directions. $D=E h^{3} / 12\left(1-v^{2}\right)$ is the flexural rigidity of the plate, $m$ represents the mass per unit area of the plate, $E$ and $v$ are respectively, Young's modulus and Poisson's ratio of the plate. 
The couples of eigenvalue/eigenmode are organized from smallest to highest eigenvalues, and to simplify notations, they are written with only one indice: $\left(\omega_{i}, \psi_{i}\right) .\left(\omega_{i}^{R}, \psi_{i}^{R}\right)$ will represent the $i$ th residual couple of eigenvalue/eigenmode.

Introducing the piezoelectric law in the equilibrium equation, and from the usual modal analysis, the modal dynamic equation is obtained in the form of (1). $b_{i j}$ represents the action of the $j$ th actuator to the $i$ th eigenmode and equals to

$$
\left.b_{i j}=\xi \int_{S} e_{31} \frac{\partial^{2} \psi_{i}}{\partial x^{2}}+e_{32} \frac{\partial^{2} \psi_{i}}{\partial y^{2}}\right) \mathrm{d} S
$$

$S$ is the area of the actuator, $e_{31}$ and $e_{32}$ are the piezoelectric coefficient, $\xi=\left(2 h+h_{p}\right)$.

In the case of the sensor's output equation, there exist two basic approaches for calculating the piezoelectric sensor voltage [15]. One approach is to consider an open-circuit configuration in which the total surface charge is assumed to be zero and the sensor voltage is obtained by integrating the electric field over the sensor. Another approach is to consider a closed-circuit configuration in which the electric field becomes zero and the total charge on the sensor is obtained by integrating the electric displacement over the sensor area. Here, the first approach is followed.

Consider the $j$ th sensor made up of a pair of two patches. The output voltage $V_{j}^{+}$that appears between the electrodes of the patch bonded on the plate at $z=h$ can be calculated as [32]

$$
V_{j}^{+}=\frac{1}{S} \int_{S} \phi\left(h+h_{p}\right)-\phi(h) \mathrm{d} S
$$

where $S$ is the effective electrode surface, assumed equals to $S=L_{p} \times l_{p}$. In the same way, the output voltage $V_{j}^{-}$over the patch bonded at $z=-h$ is

$$
V_{j}^{-}=\frac{1}{S} \int_{S} \phi(-h)-\phi\left(-h-h_{p}\right) \mathrm{d} S
$$

Then, in order to measure only pure bending motion, the output voltage $y_{j}$ over the $j$ th sensor will be

$$
y_{j}=\frac{V_{j}^{-}-V_{j}^{+}}{2}
$$

Combining the hypothesis of this approach (i.e. here the third components of electrical displacement equals to zero in the piezoelectric) with the piezoelectric constitutive behaviour équations gives the relation of $V_{j}^{+}$and $V_{j}^{-}$with respect to the strains modal displacement. The output voltage $y_{j}$ over the $j$ th sensor becomes

$$
y_{j}=\sum_{i=1}^{N} c_{j i} \alpha_{i}
$$

where

$$
\left.c_{j i}=\frac{1}{\varepsilon_{33} S} h_{p}\left(\frac{h_{p}}{2}+h\right) \int_{S} e_{31} \frac{\partial^{2} \psi_{i}}{\partial x^{2}}+e_{32} \frac{\partial^{2} \psi_{i}}{\partial y^{2}}\right) \mathrm{d} S
$$

Hence, using the expressions (27) and (32) of $b_{i j}$ and $c_{j i}$ in the optimization criteria (24) and (16) give

$$
\begin{aligned}
& J_{A}=\min _{i=1, N} \frac{\left.\sum_{j=1}^{N_{a}} \int_{S} e_{31} \frac{\partial^{2} \psi_{i}}{\partial x^{2}}+e_{32} \frac{\partial^{2} \psi_{i}}{\partial y^{2}}\right) \mathrm{d} S}{\left.\max _{A_{1}, \ldots, A_{N a}} \sum_{j=1}^{N_{a}} \int_{S} e_{31} \frac{\partial^{2} \psi_{i}}{\partial x^{2}}+e_{32} \frac{\partial^{2} \psi_{i}}{\partial y^{2}}\right) \mathrm{d} S}-\max _{i=1, N R} \frac{\left.\sum_{j=1}^{N_{a}} \int_{S} e_{31} \frac{\partial^{2} \psi_{i}}{\partial x^{2}}+e_{32} \frac{\partial^{2} \psi_{i}}{\partial y^{2}}\right) \mathrm{d} S}{\left.\max _{A_{1}, \ldots, A_{N a}} \sum_{j=1}^{N_{a}} \int_{S} e_{31} \frac{\partial^{2} \psi_{i}}{\partial x^{2}}+e_{32} \frac{\partial^{2} \psi_{i}}{\partial y^{2}}\right) \mathrm{d} S} \\
& J_{S}=\min _{i=1, N} \frac{\left.\sum_{j=1}^{N_{s}} \int_{S} e_{31} \frac{\partial^{2} \psi_{i}}{\partial x^{2}}+e_{32} \frac{\partial^{2} \psi_{i}}{\partial y^{2}}\right) \mathrm{d} S}{\left.\max _{S_{1}, \ldots, S_{N_{S}}} \sum_{j=1}^{N_{S}} \int_{S} e_{31} \frac{\partial^{2} \psi_{i}}{\partial x^{2}}+e_{32} \frac{\partial^{2} \psi_{i}}{\partial y^{2}}\right) \mathrm{d} S}-\max _{i=1, N^{R}} \frac{\left.\sum_{j=1}^{N_{s}} \int_{S} e_{31} \frac{\partial^{2} \psi_{i}}{\partial x^{2}}+e_{32} \frac{\partial^{2} \psi_{i}}{\partial y^{2}}\right) \mathrm{d} S}{\left.\max _{S_{1}, \ldots, S_{N_{S}}} \sum_{j=1}^{N_{S}} \int_{S} e_{31} \frac{\partial^{2} \psi_{i}}{\partial x^{2}}+e_{32} \frac{\partial^{2} \psi_{i}}{\partial y^{2}}\right) \mathrm{d} S}
\end{aligned}
$$

As soon as actuators and sensors are made with the same piezoelectric material, the two optimization problems are similar and results will be identical.

Note that in this work, the orientation of devices (actuators and sensors) are taken into account in the optimization process. As devices are not necessarily parallel to the plate, in order to take into account the orientation of the devices, the following transformation of variables must be applied to calculate the integrals in (33) and (34) (see Fig. 1):

$$
\begin{aligned}
& X=\left(x-x_{G}\right) \cos \theta+\left(y-y_{G}\right) \sin \theta \\
& Y=\left(y-y_{G}\right) \cos \theta-\left(x-x_{G}\right) \sin \theta
\end{aligned}
$$

$(X, Y),(x, y)$ and $\left(x_{G}, y_{G}\right)$ are respectively, the local coordinates in the referential of the device, the global coordinates in the referential of the plate and the coordinates of the device's center $G$ in the referential of the plate. 
To allow the rotation of patches near the edges of the plate, the admissible area for the center of devices is restricted to the internal rectangle presented in Fig. 1. $d$ equals to $\sqrt{l_{p}^{2}+L_{p}^{2}} / 2$.

In all simulations, the GA parameters are as follows: the population size, the crossover probability, the mutation probability and the number of generations are set as 16,100 percent, 10 percent, 50 , respectively, and at each iteration, the best parent is kept in the new generation.

\subsection{First application: tests 1 and 2: use and validation of $G A$}

In this subsection, two first examples are presented to demonstrate the feasibility and efficiency of the proposed method. The plate is discretized in $20 \times 20$ elements, each element representing a candidate location for piezoelectric patches.

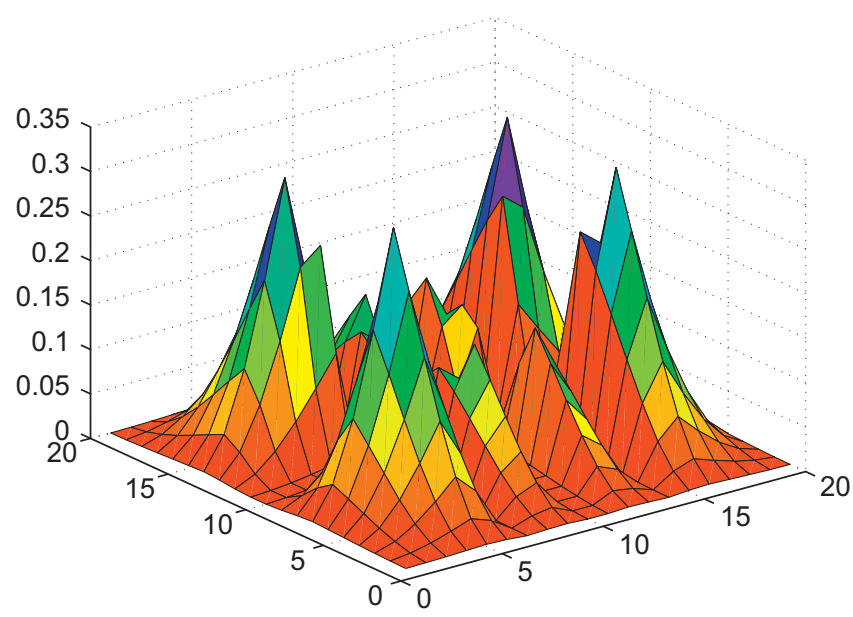

Fig. 2. Test 1: variation of criterion $J_{S}$ for one piezoelectric sensor $(\gamma=0)$.

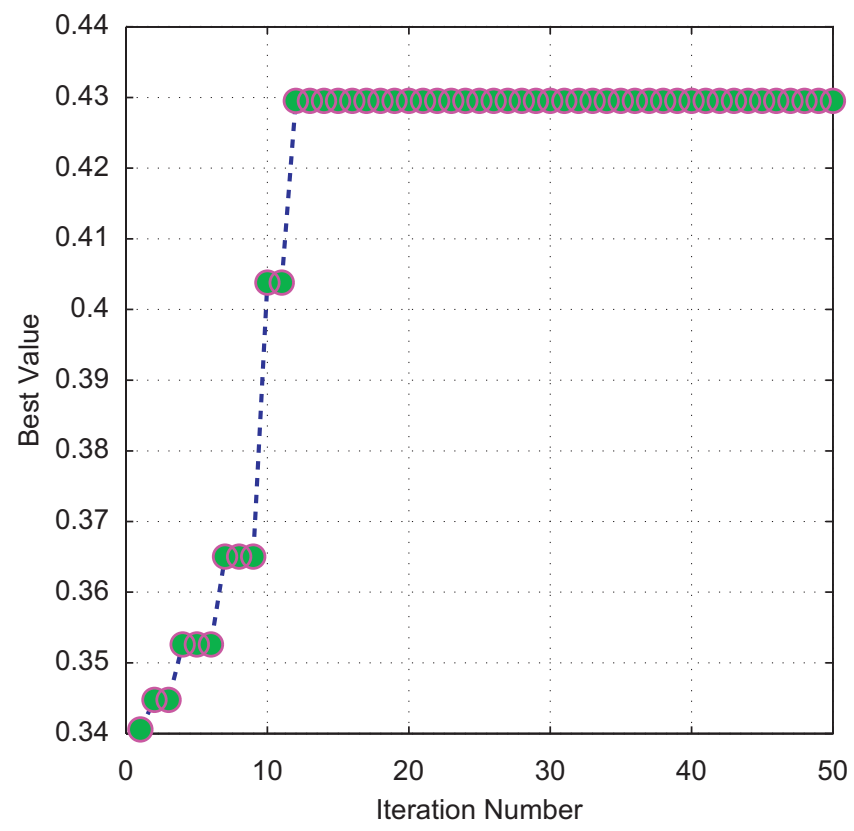

Fig. 3. Test 1: evolution of the fitness function values. 
At first GA is used to find the location of two piezoelectric sensors in order to well observe the first five vibration modes. Residual modes are neglected $\left(\gamma=0\right.$ in the criterion), and the optimization variables are restrained to $S_{j}=\left(x_{G}^{j}, y_{G}^{j}\right)$ for the two sensors. The variation of the criterion, function of the location of a single sensor, is shown in Fig. 2 . The criterion is obviously not convex and includes several local and global optima.

The convergence of the fitness function is shown in Figs. 3 and 4. The optimal obtained locations are presented in Fig. 5 and are $(0.1425,0.0825),(0.0665,0.1875)$. An exhaustive research of the global optimal locations $\left(400^{2}\right.$ configurations) leads to 32 optimal results, which include the location found by the GA. The value of the criteria is 0.429 . It means that each eigenmode is at least observed 42 percent comparing the case with one sensor solely optimally located for this eigenmode.

In a second test, the optimal locations of three sensors are studied to well observe the eight first eigenmodes. The locations $(0.0475,0.1725),(0.1805,0.0375)$ and $(0.2375,0.1875)$ are obtained using GA, with the criterion equals to 0.343 . In order to verify that these locations are local optima, the criterion is calculated for every placement near of the obtained locations (see Fig. 6). Considering for one sensor, 8 locations near its optimal location, $8^{3}$ configurations are

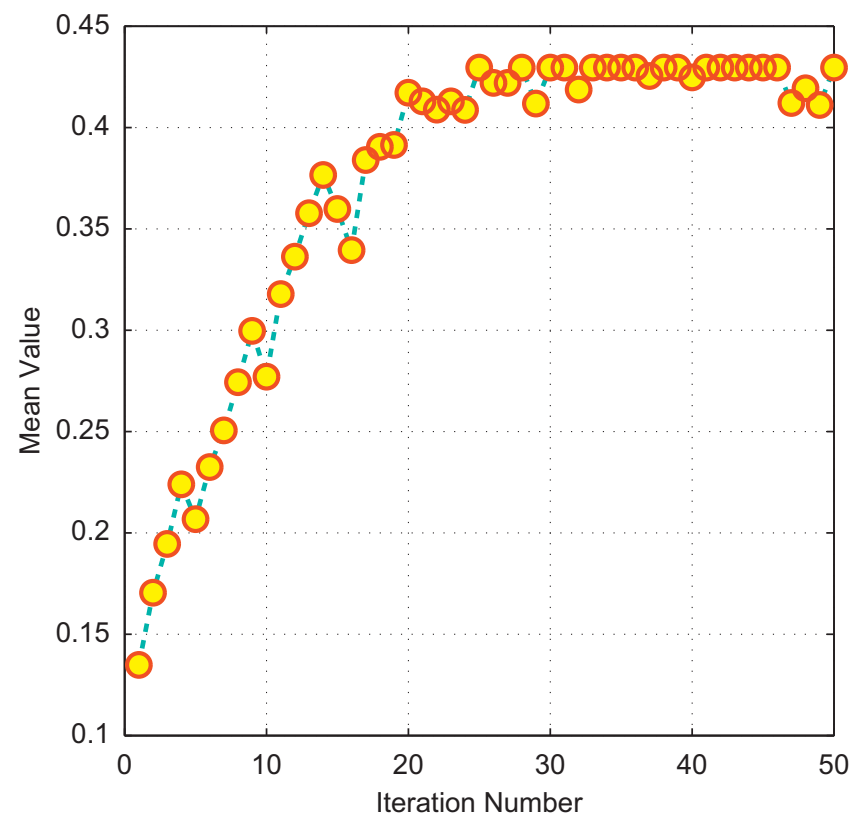

Fig. 4. Test 1: evolution of the mean value of the fitness function.

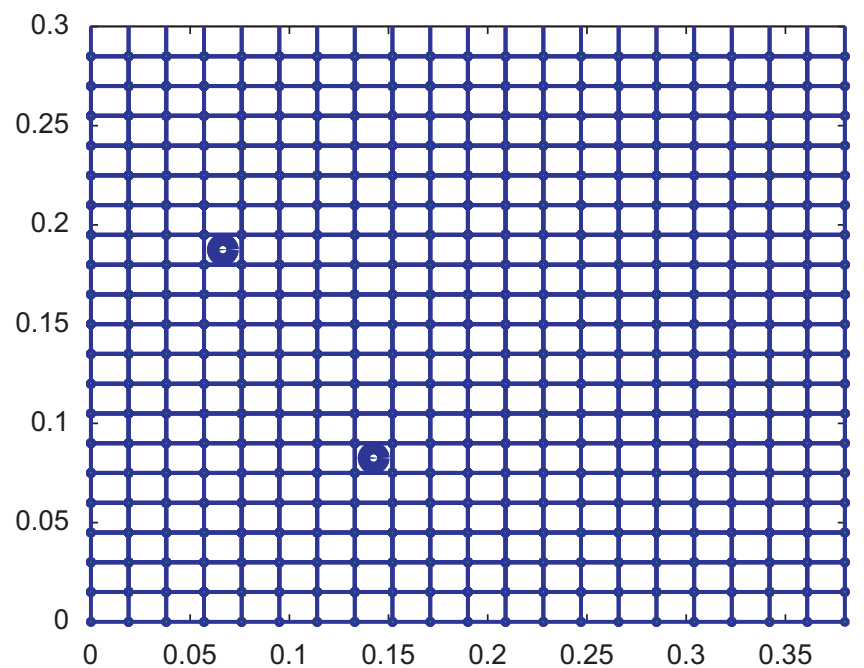

Fig. 5. Test 1: optimal location for the two piezoelectric sensors. 


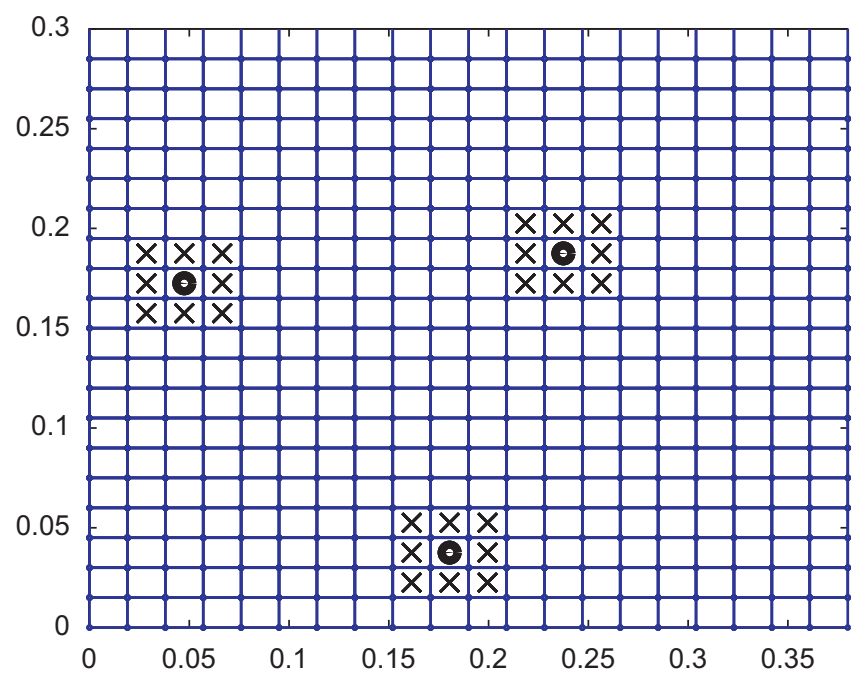

Fig. 6. Test 2: bullet: optimal locations for the three piezoelectric sensors, cross: the studied neighborhood locations.

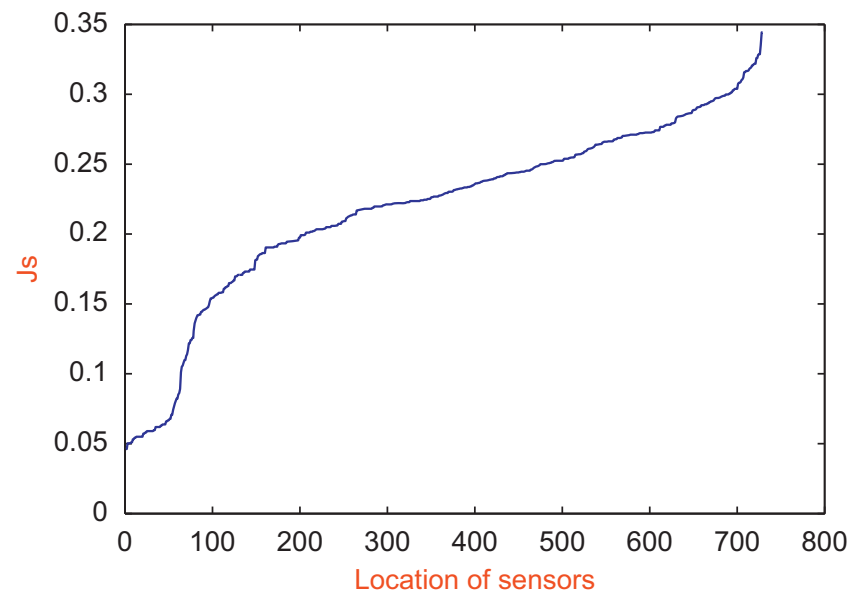

Fig. 7. Test 2: values of the criteria for each configuration, near the optimal locations.

considered. The values of the criterion are plotted Fig. 7 with increasing order. The maximal value equals to the value which corresponds to the optimal locations. This study shows that the optimal location given by GA is at least local optima.

\subsection{Second application: test 3: the use of residual eigenmodes in the criteria}

The optimization of piezoelectric actuators location is now considered and the plate is discretized in $20 \times 20$ elements. The optimization variables are again restrained to $A_{i}=\left(x_{G}^{i}, y_{G}^{i}\right)$ for the $i$ th device.

In test $3, \tilde{\gamma}=0.5$ and the number of actuators varies from 1 to 5 . The objective is to well control the 10 first eigenmodes $(N=10)$ and avoid the control of the 11th to 15th eigenmodes $\left(N^{R}=5\right)$. The better configuration should be:

- to maximize $\lambda^{U}=\left\{\min _{i=1, N}\left(\mathbf{W}_{c}^{U}\left(A_{1}, \ldots, A_{N_{a}}\right)\right)_{i i} / \max _{A_{1}, \ldots, A_{N_{a}}}\left(\mathbf{W}_{c}^{U}\left(A_{1}, \ldots, A_{N_{a}}\right)\right)_{i i}\right\}$;

- to minimize $\lambda^{R}=\left\{\max _{i=1, N^{R}}\left(\mathbf{W}_{c}^{R}\left(A_{1}, \ldots, A_{N_{a}}\right)\right)_{i i} / \max _{A_{1}, \ldots, A_{N_{a}}}\left(\mathbf{W}_{c}^{R}\left(A_{1}, \ldots, A_{N_{a}}\right)\right)_{i i}\right\}$.

The GA is used for several actuators number. Fig. 8 represents $\lambda^{U}$ and $\lambda^{R}$ with respect to $N_{a}$. These curves can be compared with results obtained without taking into account the residual modes, and shown in Fig. 9. In this case, for all values of $N_{a}$, some residual modes are better actuated than some of the $N$ first modes: the actuators are more 


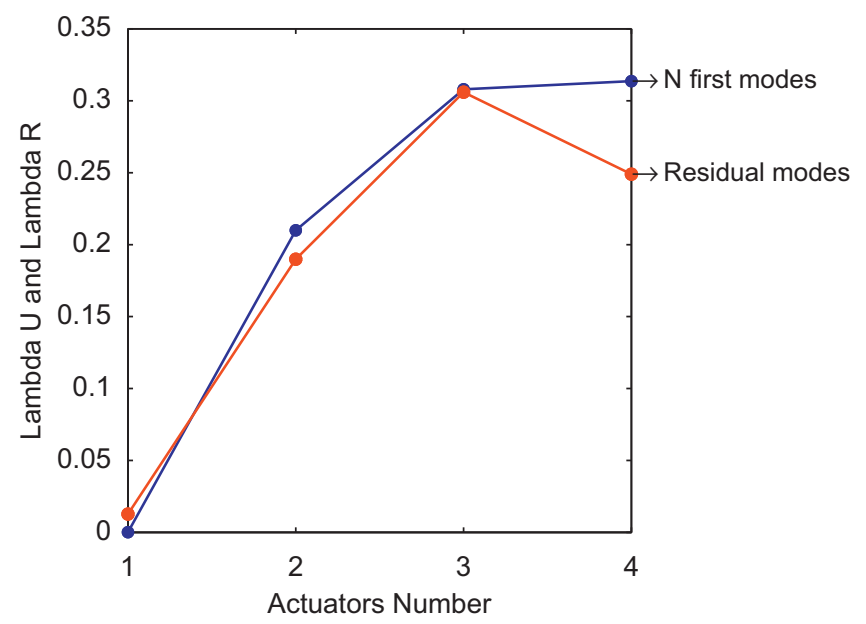

Fig. 8. Test 3: variation of $\lambda^{U}$ and $\lambda^{R}$ with $\tilde{\gamma}=0.5$.

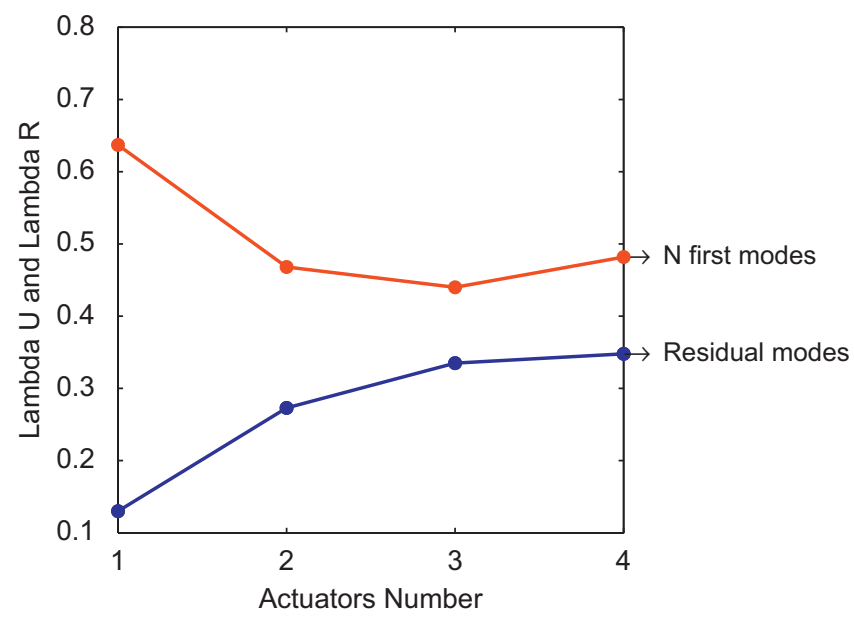

Fig. 9. Test 3: variation of $\lambda^{U}$ and $\lambda^{R}$ with $\tilde{\gamma}=0$.

efficient to actuate some residual modes than the eigenmodes which should be controlled. This result reverses when residual modes are used in the criteria. In this case, the worst controlled eigenmode between the $N$ first modes is better controlled than the best actuated residual mode: the actuation of devices is mainly on the $N$ first modes.

\subsection{Third application: tests 4 and 5: the use of the orientation of devices as an optimization variable in the criteria}

Now the orientation $\theta$ of each piezoelectric device is used as optimization variable in the GA. Here, $N=5, N^{R}=3$, $\gamma=\tilde{\gamma}=0.5$. The admissible area is discretized in $21 \times 21$ elements, each corner representing a candidate location of $G^{j}$. The admissible values of $\theta$ considered in GA are $(i-1) \pi / 12$ for $i=1, \ldots, 12$.

In test 4 , two small sensors are located (same size as the previous tests). The optimal solutions are $(0.2967,0.1775, \pi / 2)$, $(0.2611,0.2189,5 \pi / 6)$ and are plotted in Fig. 10. In order to study the influence of orientation of each sensor on the criteria, the criteria is calculated for the following configurations:

- sensor 1 is fixed and the orientation of sensor 2 is varying between 0 and $11 \pi / 12$;

- sensor 2 is fixed and the orientation of sensor 1 is varying between 0 and $11 \pi / 12$.

In these two cases, the maximal relative variation is obtained from

$$
\frac{\max _{\theta} J_{S}-\min _{\theta} J_{S}}{\max _{\theta} J} 100
$$


Their values are smaller than 0.25 percent: in this example, the use of orientations as optimization variables is not significant.

In test 5 , one long actuator is considered (see Table 1 for dimensions). The optimal location is $(0.0984,0.195, \pi / 4)$. The variation of the criteria $J_{A}$, the contribution of the $N$ first modes $\lambda^{U}$, and the residual modes $\lambda^{R}$, are plotted in Fig. 11 . The value of $\lambda^{U}$ is over 0.25 for all orientations and its maximal value is obtained when $\theta=\pi / 4$. The variation of $\lambda^{R}$ with respect to $\theta$ is more important: its value is between 0.22 to 0.32 . The minimization of $\lambda^{R}$ is obtained for $\theta=\pi / 3$. In this case, taking into account the orientation of the actuator allows to reduce the effects of residual modes.

\section{Application for active control}

In this section, we consider the active control of the previous plate, where the first five modes have to be controlled and modes 6-8 are considered residual. The external loads applied to the structure are initial conditions, dirac and sinusoidal loads. In order to decrease the vibrations, the plate is equipped with two piezoelectric actuators and sensors. Using the GA with $\gamma=\tilde{\gamma}=0.5$, these actuators and sensors are respectively, located in: $(0.2615,0.0806, \pi / 6),(0.0827,0.1222,7 \pi / 12)$, and $(0.2973,0.1778, \pi / 2),(0.2615,0.2194,5 \pi / 6)$.

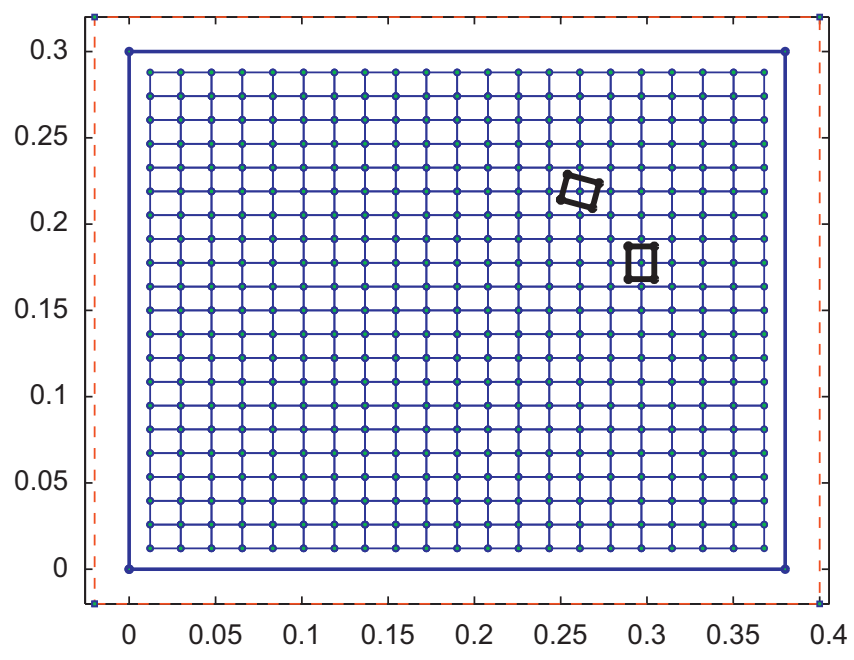

Fig. 10. Test 4: optimal location of two sensors.

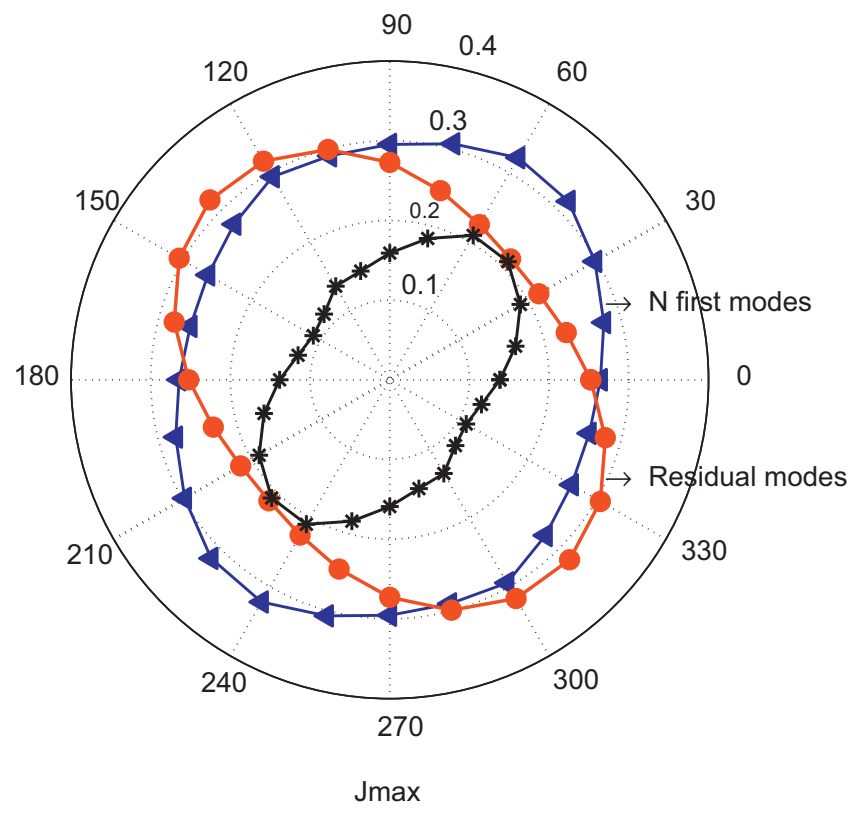

Fig. 11. Test 5: variation of $\lambda^{U}$ (triangles), $\lambda^{R}$ (circles) and $J^{A}$ (stars) with respect to $\theta$. 


\subsection{Control law and observer}

In order to actively control vibrations, a linear quadratic control method, including a state observer, is developed from the state equation (5) considering the $N$ first eigenmodes. Assuming that the state equation is controllable, it consists in using a control law:

$$
\{\boldsymbol{\phi}\}=-[\mathbf{K}]\{\mathbf{x}\}
$$

which minimizes a cost function given by

$$
J_{\phi}=1 / 2 \int_{0}^{\infty}\left[\{\mathbf{x}\}^{\mathrm{T}}[\mathbf{Q}]\{\mathbf{x}\}+\{\boldsymbol{\phi}\}^{\mathrm{T}}[\mathbf{R}]\{\boldsymbol{\phi}\}\right] \mathrm{d} t
$$

$[\mathbf{R}]$ is a positive matrix and $[\mathbf{Q}]$ is a positive semidefinite matrix. The optimal solution is

$$
[\mathbf{K}]=[\mathbf{R}]^{-1}[\mathbf{B}]^{\mathrm{T}}[\mathbf{P}]
$$

where $[\mathbf{P}]$ satisfies the Riccati equation:

$$
[\mathbf{A}]^{\mathrm{T}}[\mathbf{P}]+[\mathbf{P}][\mathbf{A}]-[\mathbf{P}][\mathbf{B}][\mathbf{R}]^{-1}[\mathbf{B}]^{\mathrm{T}}[\mathbf{P}]+[\mathbf{Q}]=0
$$

The choice of $[\mathbf{Q}]$ and $[\mathbf{R}]$ is not easy [33]. In this application, [Q] is chosen so that $\{\mathbf{x}]^{\mathrm{T}}[\mathbf{Q}]\{\mathbf{x}\}$ represents the mechanical energy. [R] is a diagonal matrix, which components are chosen such that the maximal values of $\{\boldsymbol{\phi}\}$ are less than the maximal admissible values for the piezoelectric materials under consideration.

In order to be implemented, the optimal state control law needs knowledge of the state vector $\{\mathbf{x}\}$. This knowledge is not complete since only the output voltages in $\{\mathbf{y}\}$ are observed. Assuming that the state system verifies the observability criteria, an estimation $\{\hat{x}\}$ is computed using a Luenberger observer [34] which is such that

$$
\frac{\mathrm{d}}{\mathrm{d} t}\{\hat{x}\}=[\mathbf{A}]\{\hat{x}\}+[\mathbf{B}]\{\boldsymbol{\phi}\}+[\mathbf{L}](\{\mathbf{y}\}-[\mathbf{C}]\{\hat{x}\})
$$

where $[\mathbf{L}]$ is the observance gain matrix. It is chosen such that the real parts of the eigenvalues of $[\mathbf{A}]-[\mathbf{L}][\mathbf{C}]$ are negative. Consequently, the control law applied to the actuators becomes

$$
\{\boldsymbol{\phi}\}=-[\mathbf{K}]\{\hat{x}\}
$$

\subsection{Active control of the plate subjected for several external loads}

In this subsection, the efficiency of the active control and of the optimization procedure are evaluated for several external loads. The construction of the control and observer is done using MATLAB. The three tests are described below:

- test 6: the following initial conditions are considered to excite the plate:

$$
\begin{gathered}
\alpha_{i}(t=0)=1, \quad i=1,2,3 \\
\alpha_{i}(t=0)=0 \quad \text { else }
\end{gathered}
$$

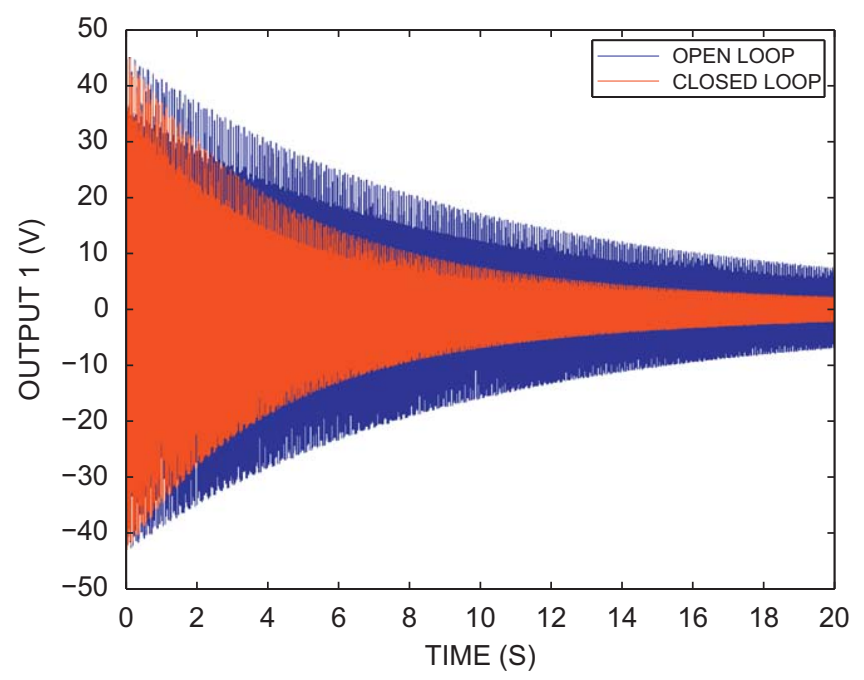

Fig. 12. Test 6: the output of sensor 1 . 


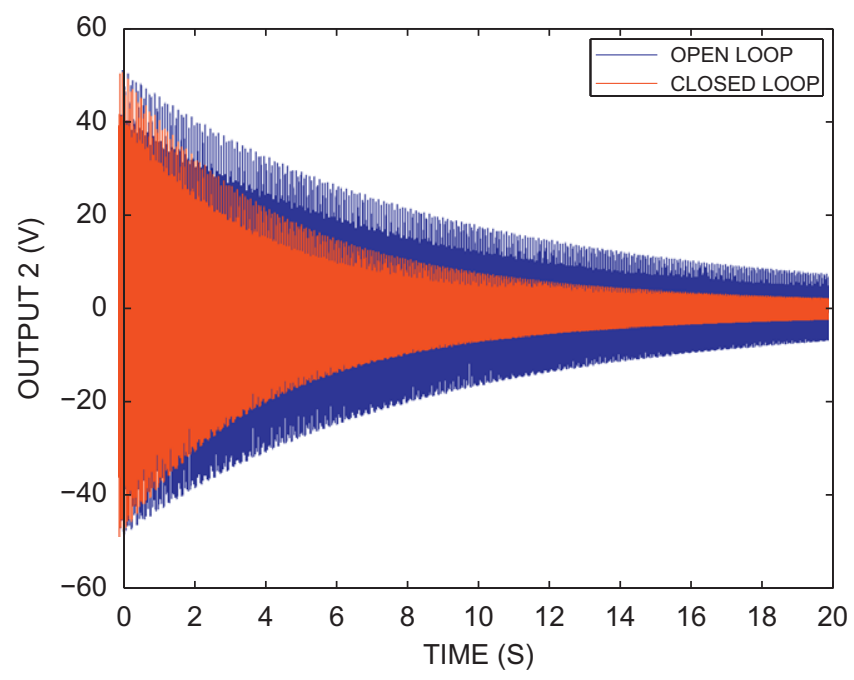

Fig. 13. Test 6: the output of sensor 2 .

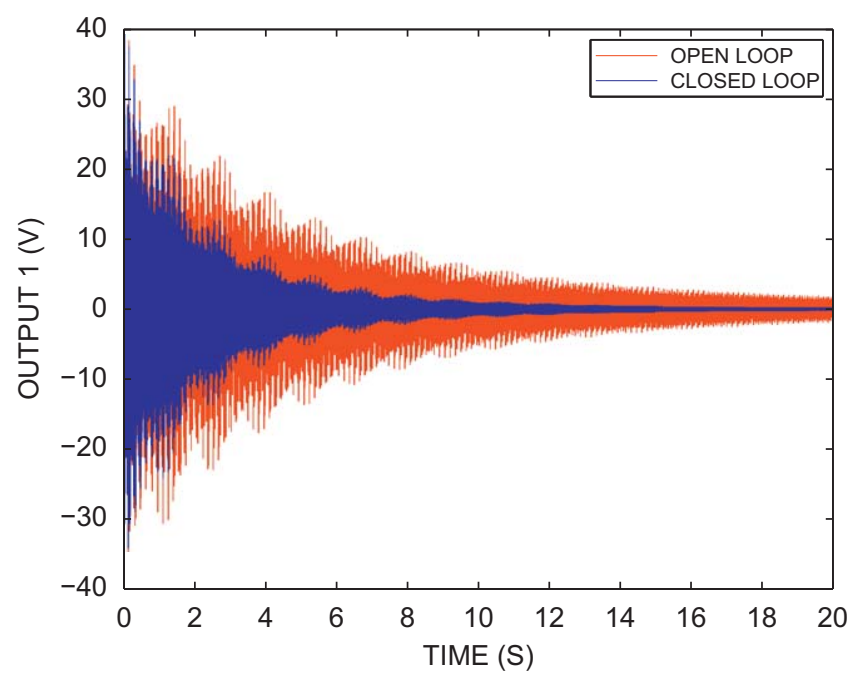

Fig. 14. Test 7: the output of sensor 1.

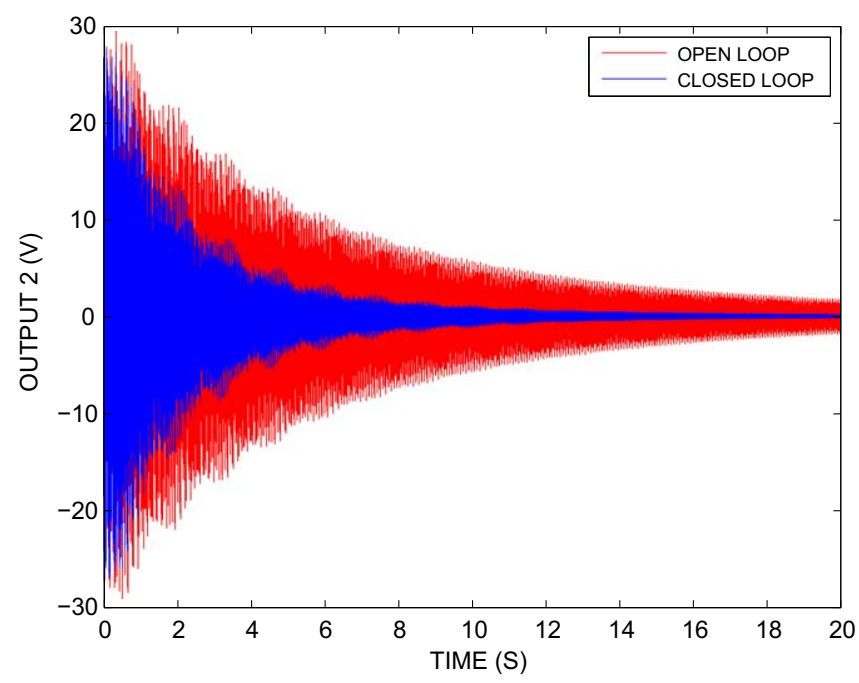

Fig. 15. Test 7: the output of sensor 2 . 


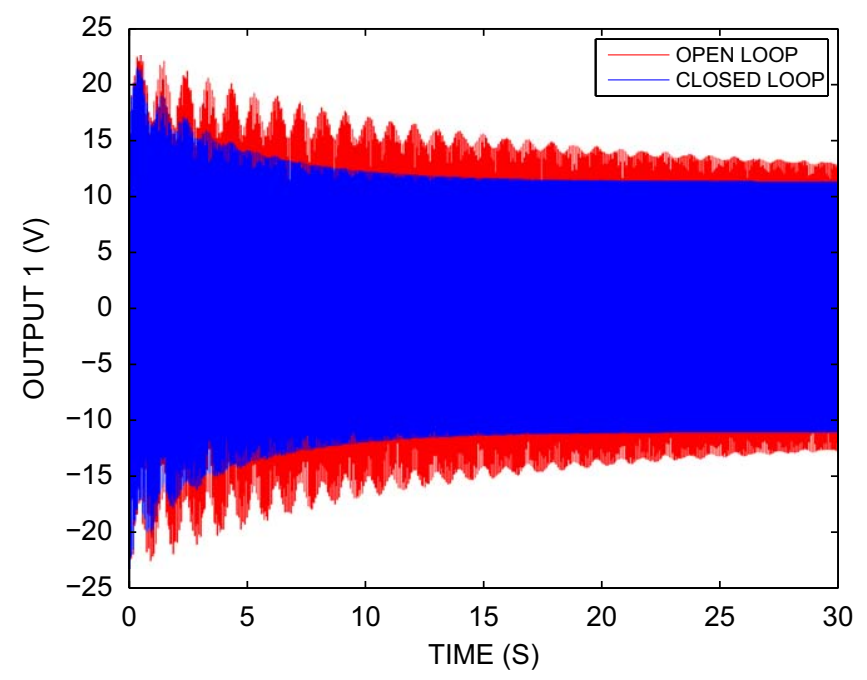

Fig. 16. Test 8: the output of sensor 1 .

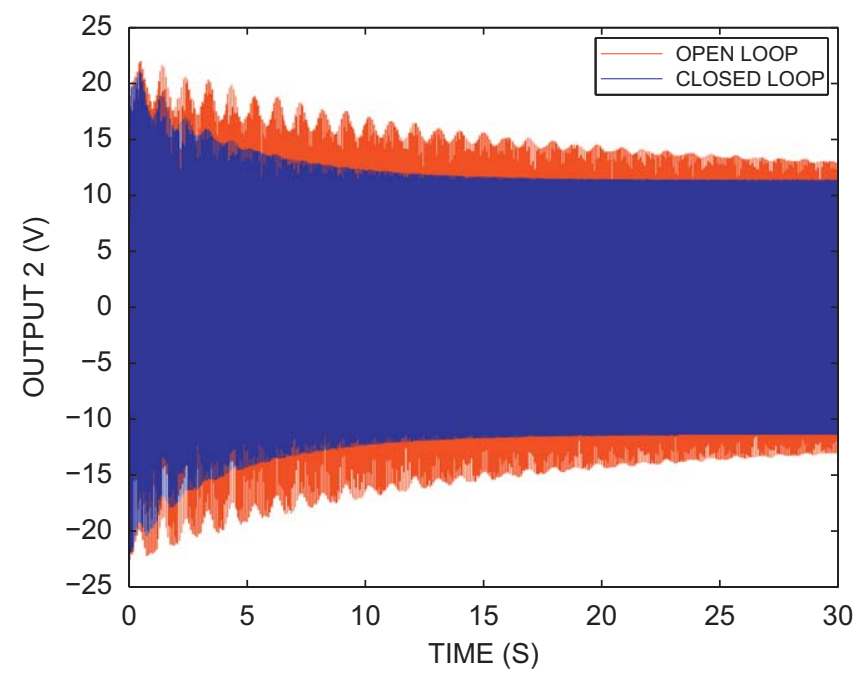

Fig. 17. Test 8: the output of sensor 2 .

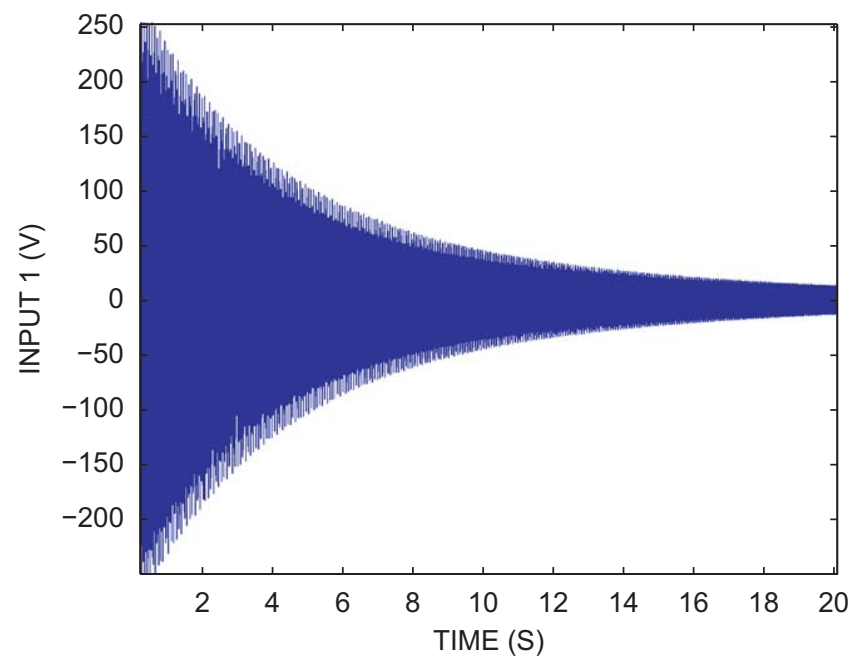

Fig. 18. Test 6: the input of actuator 1 . 


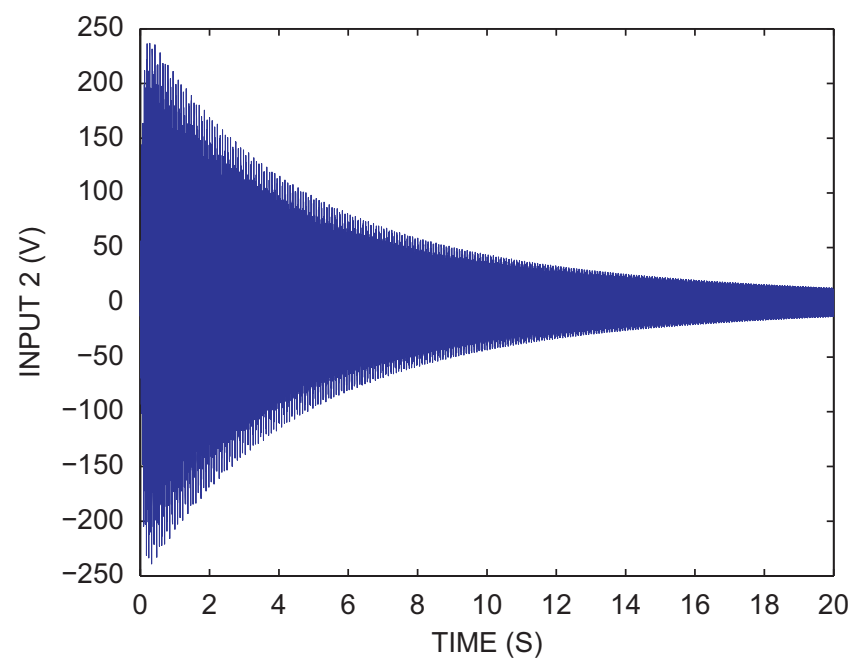

Fig. 19. Test 6: the input of actuator 2 .

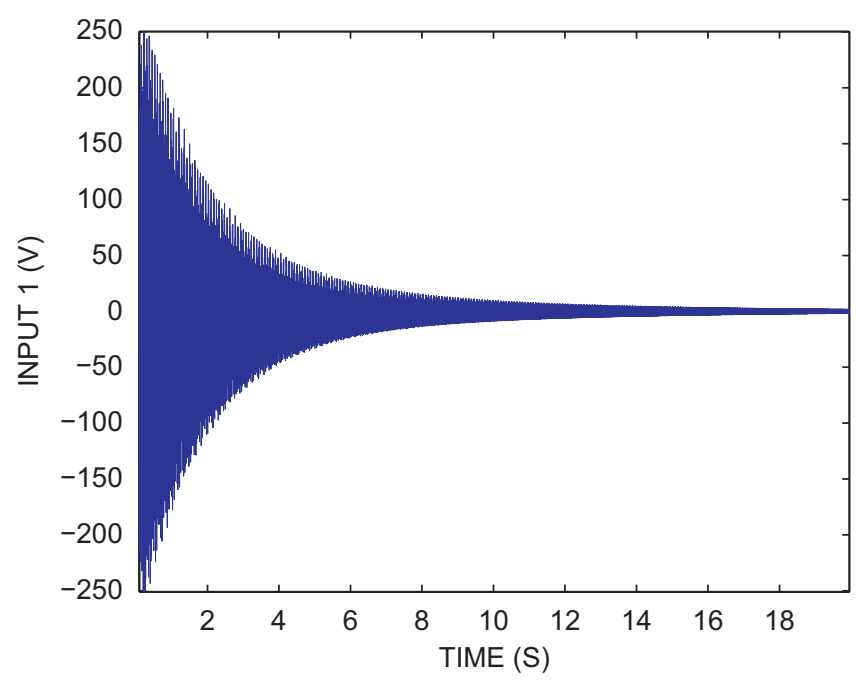

Fig. 20. Test 7: the input of actuator 1.

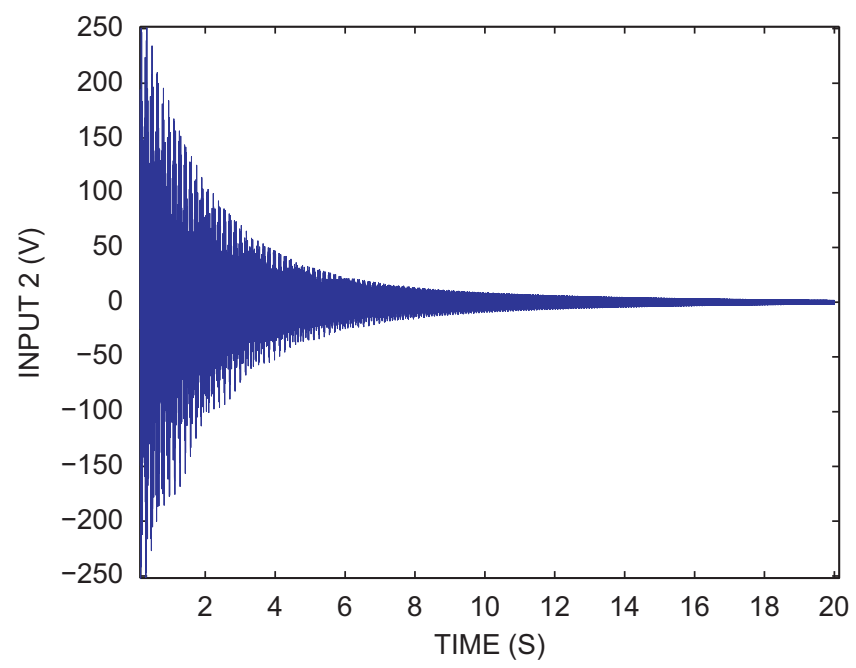

Fig. 21. Test 7: the input of actuator 2 . 


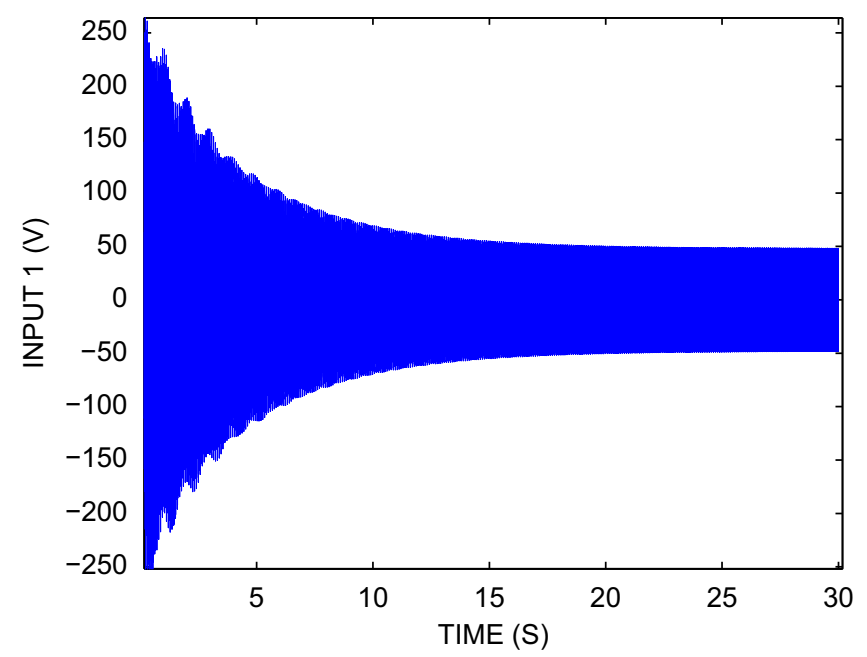

Fig. 22. Test 8: the input of actuator 1 .

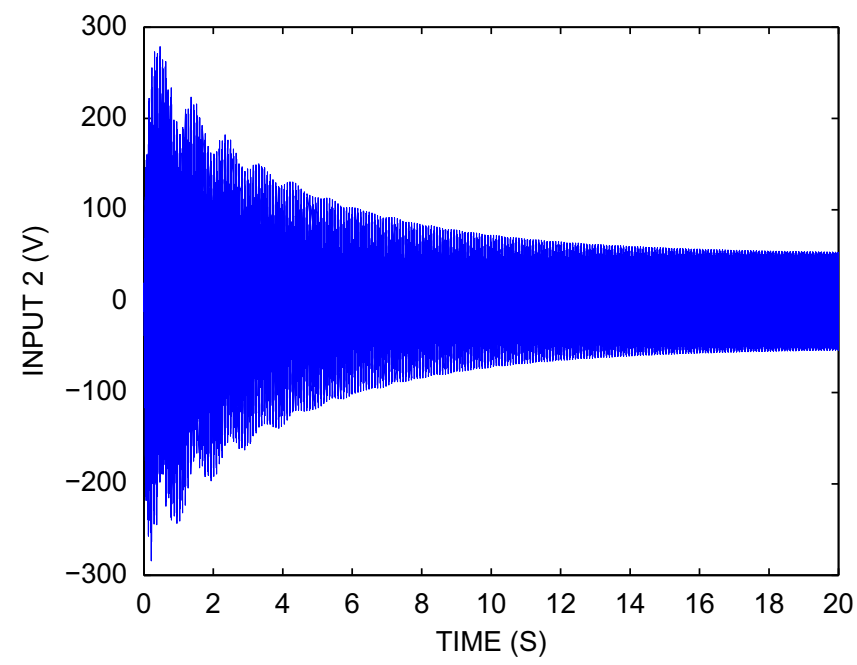

Fig. 23. Test 8: the input of actuator 2 .

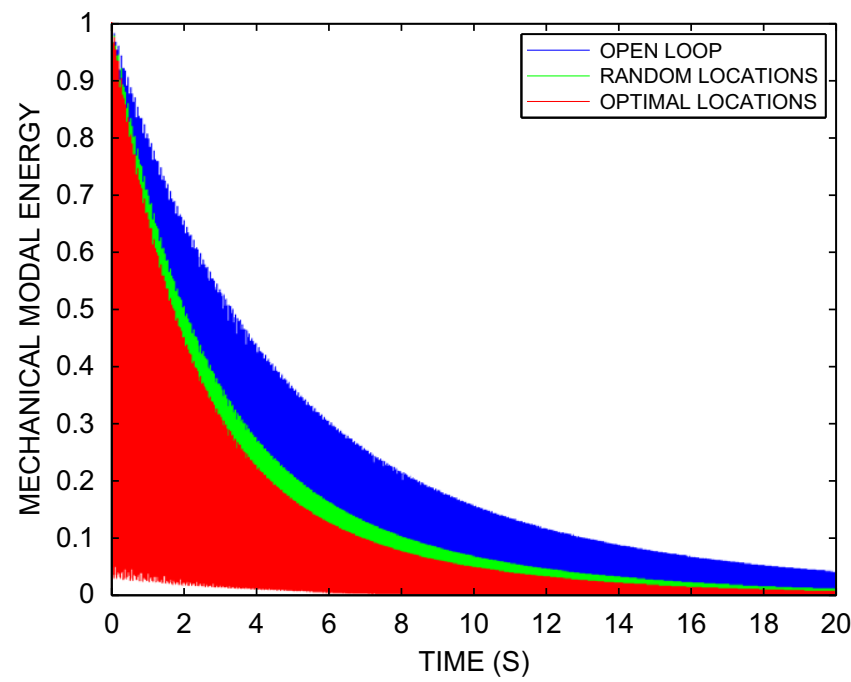

Fig. 24. Test 6: the mechanical modal energy. 
- test 7: the plate is subjected to a transversal dirac effort, located at the point $(0.05,0.06)$,

- test 8: the plate is subjected to a transversal sinusoidal effort at the point $(0.1,0.1)$ equals to $100 * \cos (200 \pi t)$.

The sensors output in open and closed loop are plotted in Figs. 12-17. The amplitude decay of the open loop response comes from natural damping, while that of the closed loop system comes mainly from the feedback control. Figs. 18-23 show the required input voltage for the active control. For each test, $[\mathbf{R}]$ is chosen such that the maximal values of the input voltage will be less or equal to $250 \mathrm{~V}$.

These results are compared with those obtained with piezoelectric actuators and sensors located randomly respectively, in $(0.0648,0.1916,0),(0.3331,0.1361,0)$, and $(0.3509,0.2055,0),(0.2055,0.1084,0)$. Figs. $24-26$ show the mechanical modal energy (normalized by the maximal value) defined by

$$
E m(t)=\frac{1}{2} \sum_{i=1}^{N+N^{R}} \dot{\alpha}_{i}^{2}(t)+\omega_{i}^{2} \alpha_{i}^{2}(t)
$$

The mechanical displacement at the middle of the plate is plotted in Figs. 27-29. For each load, the vibration of the structure with random locations decays slower than that of optimal locations obtained with GA. The simulation results show that the placement is effective and the vibration can be suppressed quickly.

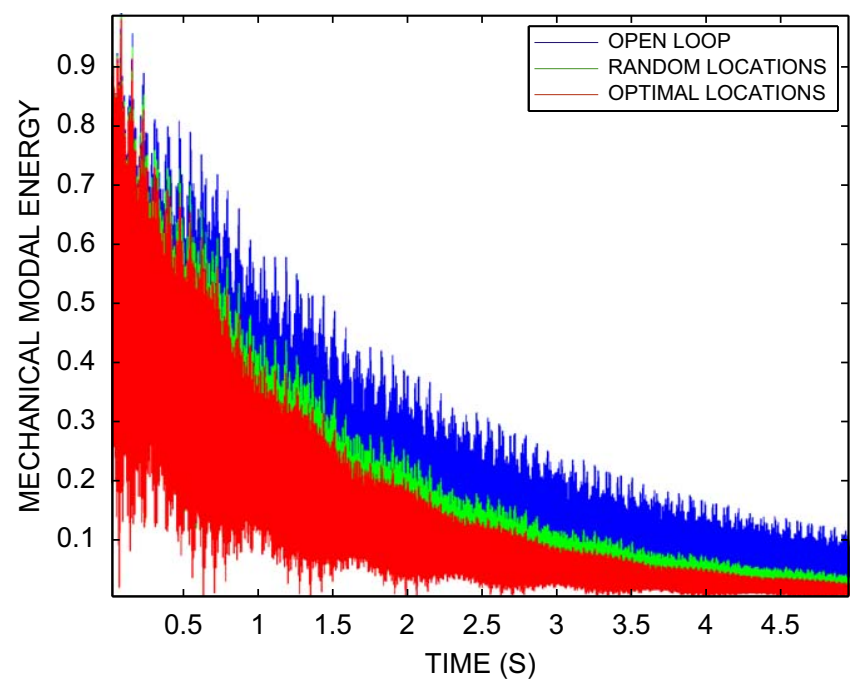

Fig. 25. Test 7: the mechanical modal energy.

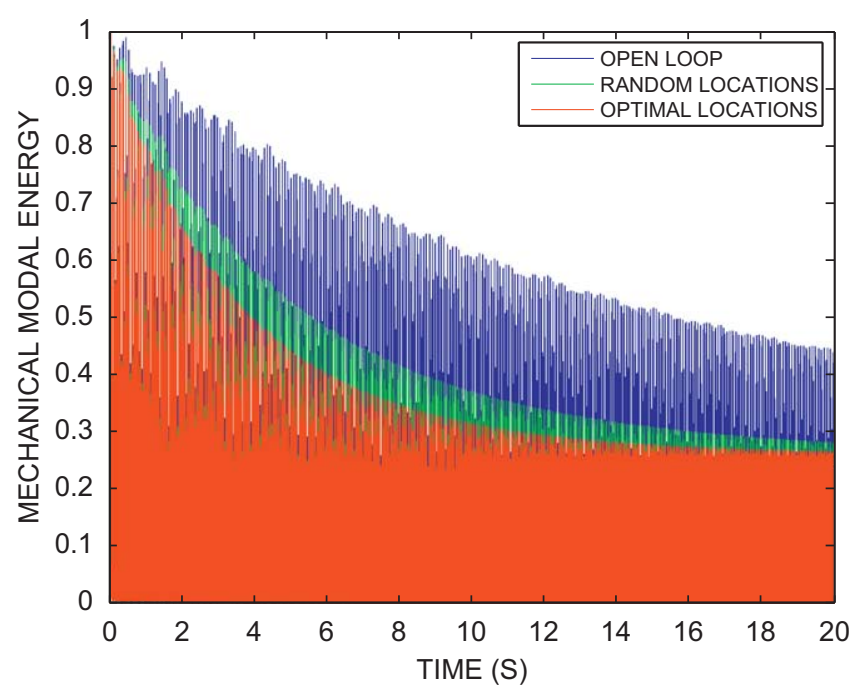

Fig. 26. Test 8: the mechanical modal energy. 


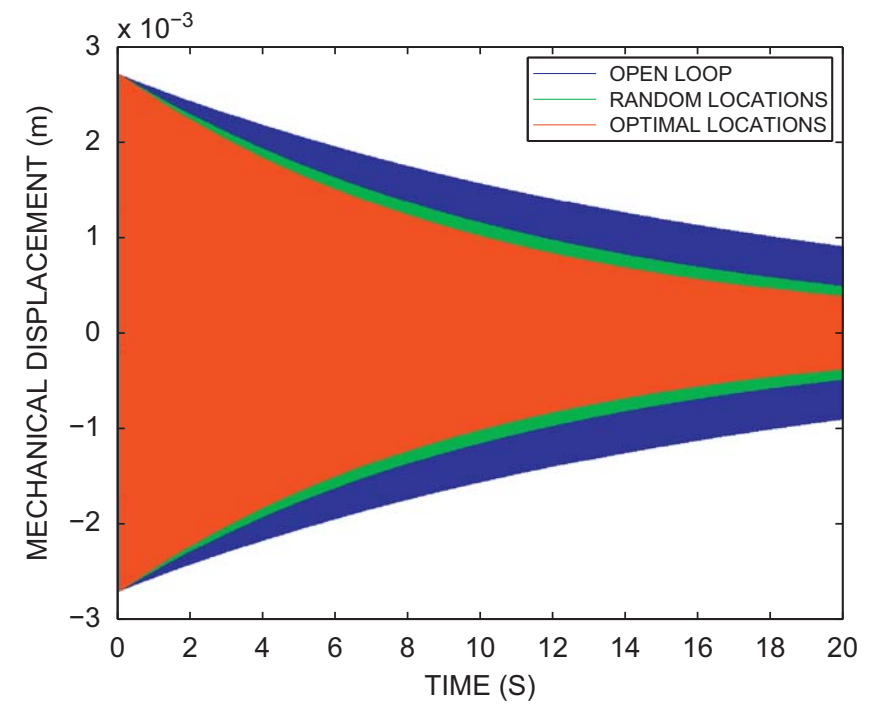

Fig. 27. Test 6: the mechanical displacement.

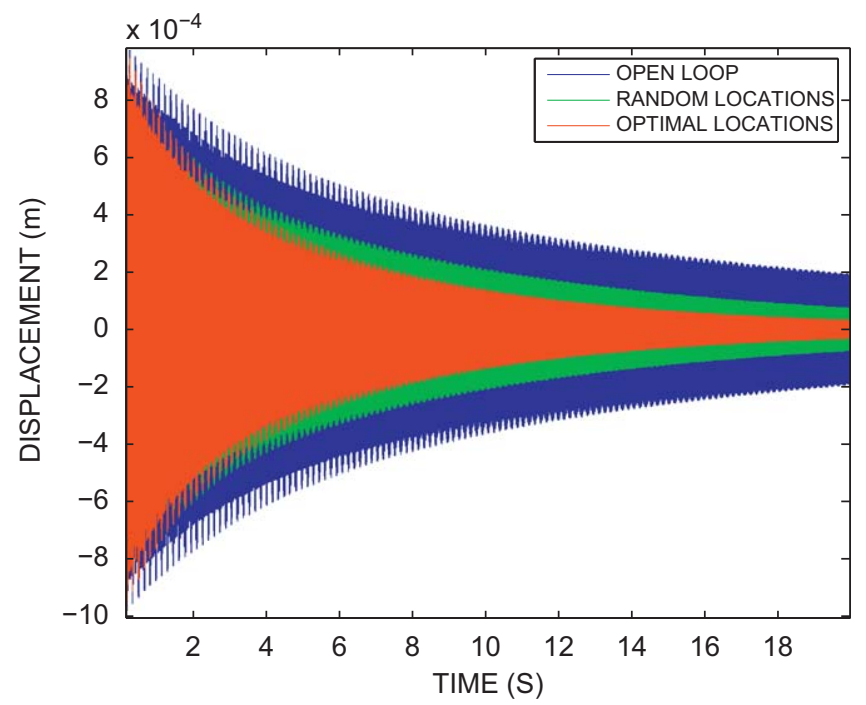

Fig. 28. Test 7: the mechanical displacement.

\section{Conclusion}

In this paper, the optimal position and orientation of piezoelectric actuators and sensors for active vibration control is considered. For each optimization problem, a modified optimization criterion is used. It derives from usual approaches, ensuring good observability and controllability of each mode of the structure and taking into account the residual modes which should be less observable and controllable. Genetic algorithms are well adapted to solve these optimization problems where the criteria are not convex and not easily derivable. Several applications are presented in the case of a simply supported plate. Simulations show the efficiency of GA for these optimization problems. The use of GA allows to take into account easily the study of the piezoelectric devices' orientation in the optimization process. The effect of this orientation is not really significant for a simply supported plate. But the introduction of this methodology with Finite Element modelling will allow to study more complex structures, where the influence of patches' orientation would be significant. 


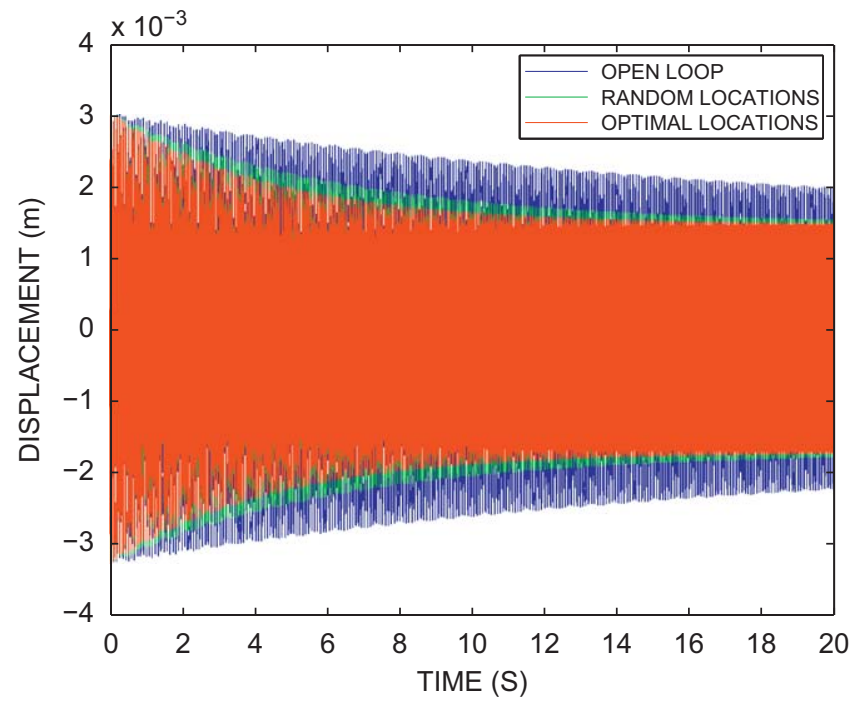

Fig. 29. Test 8: the mechanical displacement.

\section{References}

[1] M. Frecker, Recent advances in optimization of smart structures and actuators, Journal of Intelligent Material Systems and Structures 14 (2003) 207-215.

[2] S. Kondoh, C. Yatomi, K. Inoue, The positioning of sensors and actuators in the vibration control of flexible systems, JSME International Journal Serie 3 33 (2) (1990) 145-152.

[3] S. Yang, Y. Lee, Optimization of noncollocated sensor/actuator location and feedback gain in control systems, Smart Materials and Structures 2 (1993) 96-102.

[4] A. Dhingra, B. Lee, Multiobjective design of actively controlled structures using a hybrid optimization method, International Journal for Numerical Methods in Engineering 38 (1995) 3383-3401.

[5] C. Nam, Y. Kim, T. Weisshaar, Optimal sizing and placement of piezo-actuators for active flutter suppression, Smart Materials and Structures 5 (1996) $2216-2224$.

[6] I. Bruant, G. Coffignal, F. Léné, M. Vergé, A methodology for determination of piezoelectric actuator and sensor location on beam structures, Journal of Sound and Vibration 243 (5) (2001) 862-882.

[7] K. Ramesh Kumar, S. Narayanan, Active vibration control of beams with optimal placement of piezoelectric sensors/actuator pairs, Smart Materials and Structures 17 (2008).

[8] Y. Yang, Z. Jin, C. Kiong So, Integrated optimal design of vibration control system for smart beams using genetic algorithms, Journal of Sound and Vibration 282 (2005) 1293-1307.

[9] W. Liu, Z. Hou, M.A. Demetriou, A computational scheme for the optimal sensor/actuator placement of flexible structures using spatial H2 measures, Mechanical Systems and Signal Processing 20 (2006) 881-895.

[10] K. Hiramoto, H. Doki, G. Obinata, Optimal sensor/actuator placement for active vibration control using explicit solution of algebraic Riccati equation, Journal of Sound and Vibration 229 (5) (2000) 1057-1075.

[11] M. Gney, E. Eskinat, Optimal actuator and sensor placement in flexible structures using closed-loop criteria, Journal of Sound and Vibration 312 (2007) 210-233.

[12] A. Arbel, Controllability measures and actuator placement in oscillatory systems, International Journal of Control 33 (3) (1981) $565-574$.

[13] A. Hac, L. Liu, Sensor and actuator location in motion control of flexible structures, Journal of Sound and Vibration 167 (2) (1993) $239-261$.

[14] S. Devasia, T. Meressi, B. Paden, E. Bayo, Piezoelectric actuator design for vibration suppression: placement and sizing, Journal of Guidance Control and Dynamics 16 (5) (1993) 859-864.

[15] A.K. Jha, D.J. Inman, Optimal sizes and placements of piezoelectric actuators and sensors for an inflated torus, Journal of Intelligent Material Systems and Structures 14 (2003) 563-576.

[16] T. Roy, D. Chakraborty, Optimal vibration control of smart fiber reinforced composite shell structures using improved genetic algorithm, Journal of Sound and Vibration 319 (2009) 15-40.

[17] I. Bruant, L. Proslier, Optimal location of actuators and sensors in active vibration control, Journal of Intelligent Material Systems and Structures 16 (2005) 197-206.

[18] F. Peng, A. Ng, Y.-R. Hu, Actuator placement optimization and adaptive vibration control of plate smart structures, Journal of Intelligent Material Systems and Structures 16 (2005) 263-271.

[19] Q. Wang, C. Wang, A controllability index for optimal design of piezoelectric actuators in vibration control of beam structures, Journal of Sound and Vibration 242 (3) (2001) 507-518.

[20] K.D. Dhuri, P. Seshu, Piezo actuator placement and sizing for good control effectiveness and minimal change in original system dynamics, Smart Materials and Structures 15 (2006) 1661-1672.

[21] W. Gawronski, Simultaneous placement of actuators and sensors, Journal of Sound and Vibration 228 (4) (1999) $915-922$.

[22] Z.-C. Qiu, X.-M. Zhang, H.-X. Wu, H.-H. Zhang, Optimal placement and active vibration control for piezoelectric smart flexible cantilever plate, Journal of Sound and Vibration 301 (2007) 521-543.

[23] D. Halim, S.O. Reza Moheimani, An optimization approach to optimal placement of collocated piezoelectric actuators and sensors on a thin plate, Mechatronics 13 (2003) 27-47.

[24] L. Gaudiller, J. Der Hagopian, Active control of flexible structures using a minimum number of components, Journal of Sound and Vibration 193 (3) (1996) 713-741.

[25] M. Collet, Shape optimization of piezoelectric sensors dealing with spill-over instabilities, Proceedings of Active 99 (2) (1999) $1125-1134$. 
[26] Z. Michalewicz, D. Dasgupta, R. Le Riche, M. Schoenauer, Evolutionary algorithms for constrained engineering problems, Computers Engineering 30 (4) (1996) 851-870.

[27] A. Preumont, Vibration Control of Active Structures, Kluwer Academic Publishers, Dordrecht, 1999.

[28] J.-H. Han, I. Lee, Optimal placement of piezoelectric sensors and actuators for vibration control of a composite plate using genetic algorithms, Smart Materials and Structures 8 (1999) 257-267.

[29] A.M. Sadri, J.R. Wright, R.J. Wynne, Modelling and optimal placement of piezoelectric actuators in isotropic plates using genetic algorithms, Smart Materials and Structures 8 (1999) 490-498.

[30] K.H. Ip, P.-C. Tse, Optimal configuration of a piezoelectric patch for vibration control of isotropic rectangular plates, Smart Materials and Structures 10 (2001) 395-403.

[31] Y. Yang, Z. Jin, C. Kiong Soh, Integrated optimal design of vibration control system for smart beams using genetic algorithms, Journal of Sound and Vibration 282 (2005) 1293-1307.

[32] M.H. Kargarnovin, M.M. Najafizadeh, N.S. Viliani, Vibration control of a functionally graded material plate patched with piezoelectric actuators and sensors under a constant electric charge, Smart Materials and Structures 16 (2007) 1252-1259.

[33] K.K. Ang, S.Y. Wang, S.T. Quek, Weighted energy linear quadratic regulator vibration control of piezoelectric composite plates, Smart Materials and Structures 11 (2002) 98-106.

[34] T. Kailath, Linear Systems, Prentice-Hall, Englewood Cliffs, NJ, 1980. 